MATHEMATICS OF COMPUTATION

Volume 76, Number 260, October 2007, Pages 1801-1832

S 0025-5718(07)01991-6

Article electronically published on April 20, 2007

\title{
HP A-PRIORI ERROR ESTIMATES FOR A NON-DISSIPATIVE SPECTRAL DISCONTINUOUS GALERKIN METHOD TO SOLVE THE MAXWELL EQUATIONS IN THE TIME DOMAIN
}

\author{
S. PERNET AND X. FERRIERES
}

\begin{abstract}
In this paper, we present the $h p$-convergence analysis of a nondissipative high-order discontinuous Galerkin method on unstructured hexahedral meshes using a mass-lumping technique to solve the time-dependent Maxwell equations. In particular, we underline the spectral convergence of the method (in the sense that when the solutions and the data are very smooth, the discretization is of unlimited order). Moreover, we see that the choice of a non-standard approximate space (for a discontinuous formulation) with the absence of dissipation can imply a loss of spatial convergence. Finally we present a numerical result which seems to confirm this property.
\end{abstract}

\section{INTRODUCTION}

The most widely used time domain method for solving Maxwell equations is the Finite Difference Time Domain method (FD-TD) based on the well known Yee scheme [5], 6]. This method uses an orthogonal Cartesian grid and is based on a centered difference approximation in space and a leap-frog approximation in time. That provides a second order accurate scheme. However the FD-TD method suffers from a certain number of drawbacks. For example, to treat curved objects, the staircase approximation of the boundary generates parasitic diffraction phenomena which can seriously damage the accuracy of the solution [7.

Scientists and engineers have tried to develop several efficient methods which make it possible to take into account the complex shapes of the objects [25, [9]. Moreover, the growing need to solve accurately propagating electromagnetic waves over many wavelengths has forced them to develop high-order or spectral methods [27, 8].

Their first choice has naturally turned to the Finite Element Method (FEM) which is a powerful tool to develop new numerical techniques 26. One of the difficulties in using an FEM in the Maxwell types of problems is the construction of a finite dimension subspace of the continuous space $H(\operatorname{curl}, \Omega)$. Indeed, the tangential components of a function belonging to $H(\operatorname{curl}, \Omega)$ are continuous across any surface, but the normal components of the same function may be discontinuous. It is well know that the use of classical Lagrange finite elements of the space $\left[H^{1}(\Omega)\right]^{3}$ leads to spurious solutions. The appropriate finite element space was introduced by Nedelec in the 1980s [21], 22]. Unfortunately, the classical version of the edge finite elements leads to a high computational cost since a matrix inversion is needed at each time

Received by the editor June 20, 2005 and, in revised form, June 4, 2006.

2000 Mathematics Subject Classification. Primary 35B45; Secondary 65M12.

(C) 2007 American Mathematical Society Reverts to public domain 28 years from publication 
step. This drawback becomes more and more important when the order of the approximation increases. The mass-lumping technique is used in order to use this type of method in transient problems. One of the most efficient methods for solving the Maxwell equations was developed by Cohen and Monk in [23]. In this method, the use of the Gauss-Lobatto quadrature formulae yields a block diagonal mass matrix which allows one to obtain an explicit scheme for all polynomial orders of approximation.

The second choice is the use of Discontinuous Galerkin Methods (DGM). These methods were born in the first half of the Seventies throughout the work of Reed and Hill [18, on the scalar neutron transport equation. The first mathematical analysis was carried out by Lesaint and Raviart in 1974 [19. One of the basic ideas came from certain authors who weakly imposed the Dirichlet boundary condition in the FEMs instead of taking it into account directly in functional spaces. Then they decided to use this technique not only on the boundary of the computational domain but directly on the boundary of each element of the mesh in order to restore certain continuities of the solution of the studied problem (for example tangential, normal continuity, etc.). Following these first studies, many DGMs were developed and analyzed by many scientists in order to solve a large variety of problems (hyperbolic, parabolic, elliptic, etc.). An exhaustive review of these methods since their beginning is presented in 14. However, one will note that few papers deal with the resolution of the Maxwell equations. In fact the use of this type of method to solve electromagnetism problems is relatively recent. For the frequency domain, one can quote the works of [17, [16] and for the time domain, one can quote the works of [24] (space-time discontinuous approximation), 12 . (efficient local divergence-free basis functions), 15] (refinements on cartesian grid), [8] (very efficient spectral discontinuous spatial approximation with low storage Runge-Kutta scheme for time approximation : high order RKDG scheme). One can notice that before the use of these high-order methods, Finite Volume methods (that can be viewed as low order DG schemes) were used to solve the Maxwell equations. These methods suffer from the too important presence of dissipation [10] or dispersion [11] which makes their use inaccurate in problems of big size in terms of wavelength.

The DGM have the following advantages:

- arbitrary order which is chosen according to the precision on the desired exact solution.

- methods easily parallelisable: discontinuous elements, mass matrices which are diagonal per blocks (= number of degrees of freedom in the cell).

- to treat complicated geometries and simple ways to treat the boundary conditions.

- adaptive strategies: space refinements natural (without taking account of the continuities as in finite elements), order of approximation different from one cell to the others.

Moreover, there are two approaches in implementing the DGMs, namely, the $h$ version and the $p$-version. The $h$-version allows the mesh size to be decreased to achieve convergence at a rate of the employed polynomial basis. The alternative $p$ version allows the order of polynomials to be increased with the sizes of the elements kept at an initial triangulation. A hybrid $h p$-version can also be considered. This paper is devoted to the study of the convergence study of this type of method. 
The outline of the paper is as follows. In section 2, we describe the discontinuous Galerkin formulation that we have chosen to solve the Maxwell equations. In section 3 , we justify the choice of an $H^{1}$-type projector to carry out our analysis and we derive some $h p$-projection errors for this one. In section 4 , first we determine the a-priori error estimates of the DGM for the spatial semi-discrete approximation without numerical integration; second, we study the effect of the use of the Gauss quadrature rule to compute the integrals on the previous error estimates. Finally, in section 5, a numerical example which confirms the theoretical analysis is given.

\section{Presentation of the discontinuous Galerkin method}

2.1. Time-dependent Maxwell's equations. Let $\Omega$ be a bounded open subset of $\mathbb{R}^{3}$ whose boundary is $\partial \Omega$ and $\mathbf{n}$ denotes the unit outward normal to $\Omega$. Let $\underline{\underline{\varepsilon}}(x), \underline{\underline{\mu}}(x)$ and $\underline{\underline{\sigma}}(x)$ denote, respectively, the permittivity, the permeability and the conductivity tensors of the medium.

We consider the problem described by the Maxwell equations: Find $(\mathbf{E}, \mathbf{H}): \Omega \times$ ] $0, T\left[\rightarrow \mathbb{R}^{3} \times \mathbb{R}^{3}\right.$ such that:

$$
\left\{\begin{array}{c}
\underline{\underline{\varepsilon}} \frac{\partial \mathbf{E}}{\partial t}-\nabla \times \mathbf{H}+\underline{\underline{\sigma}} \mathbf{E}+\mathbf{J}_{s}=0 \text { in } \Omega, \\
\underline{\mu} \frac{\partial \mathbf{H}}{\partial t}+\nabla \times \mathbf{E}=0 \text { in } \Omega, \\
\mathbf{E} \times \mathbf{n}(x)=0 \text { on } \partial \Omega, \\
\mathbf{E}(x, 0)=\mathbf{E}_{0}(x) \text { and } \mathbf{H}(x, 0)=\mathbf{H}_{0}(x) \text { in } \Omega,
\end{array}\right.
$$

where $\mathbf{E}, \mathbf{H}$ denote the electric and magnetic field intensities, $\mathbf{J}_{s}$ specifies the applied current and $\mathbf{E}_{0}, \mathbf{H}_{0}$ are the initial conditions.

We assume that $\underline{\underline{\varepsilon}}, \underline{\mu}, \underline{\underline{\sigma}} \in\left[L^{\infty}(\Omega)\right]^{3 \times 3}$ are symmetric definite positive matrices and $\exists C_{1}, C_{2}>0$ such that:

$$
\forall \xi \in \mathbb{R}^{3}: C_{1}|\xi|^{2} \leq \underline{\underline{\varepsilon}} \xi \cdot \xi \leq C_{2}|\xi|^{2}, C_{1}|\xi|^{2} \leq \underline{\underline{\mu}} \xi \xi \xi \leq C_{2}|\xi|^{2}, C_{1}|\xi|^{2} \leq \underline{\underline{\sigma}} \xi \cdot \xi \leq C_{2}|\xi|^{2} .
$$

Moreover if we assume $\mathbf{J}_{s} \in C^{0}\left(0, T ;\left[L^{2}(\Omega)\right]^{3}\right)$, we have the existence and the uniqueness of the solution $(\mathbf{E}, \mathbf{H}) \in\left[C^{1}\left(0, T ;\left[L^{2}(\Omega)\right]^{3}\right) \cap C^{0}\left(0, T ; H_{0}(\operatorname{curl}, \Omega)\right)\right]^{2}$ [3].

2.2. Discontinuous formulation. We assume that the computational domain, $\Omega$, is split into a set of cells, $\mathscr{T}_{h}$ such that $\Omega=\bigcup_{i=1}^{N_{e}} K_{i}$, where $K_{i} \in \mathscr{T}_{h}, \dot{K}_{i} \cap \dot{K}_{j}=$ $\emptyset, \forall i \neq j$ and $K_{i}$ is a hexahedron. We denote the set of faces of $\mathscr{T}_{h}$ by $\mathscr{F}_{h}=\mathscr{F}_{h}^{i} \cup \mathscr{F}_{h}^{b}$ where $\mathscr{F}_{h}^{i}\left(\Gamma \in \mathscr{F}_{h}^{i}, \Gamma=K^{\prime} \cap K\right)$ and $\mathscr{F}_{h}^{b}\left(\Gamma \in \mathscr{F}_{h}^{b}, \Gamma=K \cap \partial \Omega\right)$ are the sets of the interior and boundary faces. To each element $K \in \mathscr{T}_{h}$, we associate the outward unit normal $\mathbf{n}_{K}$.

For a real $s \geq 0$, we define the classical broken space:

$$
H^{s}\left(\mathscr{T}_{h}\right)=\left\{v \in L^{2}(\Omega): \forall K \in \mathscr{T}_{h}, v_{\mid K} \in H^{s}(K)\right\} .
$$

$H^{s}\left(\mathscr{T}_{h}\right)$ is equipped with the natural norm: Let $v \in H^{s}\left(\mathscr{T}_{h}\right)$,

$$
\|v\|_{s, h}=\left(\sum_{K \in \mathscr{T}_{h}}\|v\|_{s, K}^{2}\right)^{\frac{1}{2}},
$$

where $\|\cdot\|_{s, K}$ is the usual Sobolev norm of $H^{s}$ on $K$. 
For $s>\frac{1}{2}$, we define the jump of a function $v \in H^{s}\left(\mathscr{T}_{h}\right)$ :

$$
\begin{gathered}
\forall \Gamma \in \mathscr{F}_{h}^{i} \text { such that } \Gamma=K^{\prime} \cap K, \llbracket v \rrbracket_{\Gamma}^{K}=\left(v_{\mid K^{\prime}}\right)_{\mid \Gamma}-\left(v_{\mid K}\right)_{\mid \Gamma} \\
\forall \Gamma \in \mathscr{F}_{h}^{b} \text { such that } \Gamma \subset \partial K, \llbracket v \rrbracket_{\Gamma}^{K}=-\left(v_{\mid K}\right)_{\mid \Gamma} .
\end{gathered}
$$

We denote $\mathbf{H}^{s}\left(\mathscr{T}_{h}\right)$ as the vectorial broken space $\left[H^{s}\left(\mathscr{T}_{h}\right)\right]^{3}$ and its norm is defined by

$$
\|v\|_{s, h}=\left(\sum_{i=1}^{3}\left\|v_{i}\right\|_{s, h}^{2}\right)^{\frac{1}{2}}
$$

where $v=\left(v_{1}, v_{2}, v_{3}\right) \in \mathbf{H}^{s}\left(\mathscr{T}_{h}\right)$.

We rewrite the problem (2.1) under the following discontinuous form:

Find $(\mathbf{E}(\cdot, t), \mathbf{H}(\cdot, t)) \in \mathbf{H}^{1}\left(\mathscr{T}_{h}\right) \times \mathbf{H}^{1}\left(\mathscr{T}_{h}\right)$ such that, $\forall K \in \mathscr{T}_{h}$ and $\forall \phi_{1}, \phi_{2} \in$ $\mathbf{H}^{1}\left(\mathscr{T}_{h}\right)$,

$$
\left\{\begin{array}{c}
\frac{d}{d t} \int_{K} \underline{\underline{\varepsilon}} \mathbf{E}_{K} \cdot \phi_{1 K} d x-\int_{K} \nabla \times \mathbf{H}_{K} \cdot \phi_{1 K} d x \\
+\int_{K} \underline{\underline{\sigma}} \mathbf{E}_{K} \cdot \phi_{1 K} d x+\int_{K} \mathbf{J}_{s} \cdot \phi_{1 K} d x \\
=\int_{\partial K} \alpha \llbracket \mathbf{n}_{K} \times\left(\mathbf{E} \times \mathbf{n}_{K}\right) \rrbracket_{\partial K}^{K} \cdot \phi_{1 K} d \sigma+\int_{\partial K} \beta \llbracket \mathbf{H} \times \mathbf{n}_{K} \rrbracket_{\partial K}^{K} \cdot \phi_{1 K} d \sigma \\
\frac{d}{d t} \int_{K} \underline{\underline{\mu}}=\mathbf{H}_{K} \cdot \phi_{2 K} d x+\int_{K} \nabla \times \mathbf{E}_{K} \cdot \phi_{2 K} d x \\
=\int_{\partial K} \gamma \llbracket \mathbf{E} \times \mathbf{n}_{K} \rrbracket_{\partial K}^{K} \cdot \phi_{2 K} d \sigma+\int_{\partial K} \delta \llbracket \mathbf{n}_{K} \times\left(\mathbf{H} \times \mathbf{n}_{K}\right) \rrbracket_{\partial K}^{K} \cdot \phi_{2 K} d \sigma
\end{array}\right.
$$

where $\mathbf{E}_{K}=\mathbf{E}_{\mid K}, \mathbf{H}_{K}=\mathbf{H}_{\mid K}, \phi_{j K}=\phi_{j \mid K}, d \sigma$ is the surface measurement associated with $\partial K$ and $\alpha, \beta, \gamma, \delta$ are four reals that could be different from one face to another.

We get a non-dissipative formulation. For that we choose the parameters :

- $\forall \Gamma \in \mathscr{F}_{h}^{i}, \alpha, \delta=0, \beta=-\frac{1}{2}$ and $\gamma=\frac{1}{2}$,

- $\forall \Gamma \in \mathscr{F}_{h}^{b}, \alpha, \delta=0, \beta=0$ and $\gamma=1$.

Indeed, by using this choice, the classical electromagnetic energy $\mathscr{E}(t)=\int_{\Omega} \underline{\underline{\epsilon}} \mathbf{E}(t)$. $\mathbf{E}(t) d \mathbf{x}+\int_{\Omega} \underline{\mu} \mathbf{H}(t) \cdot \mathbf{H}(t) d \mathbf{x}$ is time-conserved, i.e. $\mathscr{E}(t)=\mathscr{E}(0), \forall t$.

2.3. Spatial approximation. Given a non-negative integer $r$ and $E \subset \mathbb{R}^{d}, Q_{r}(E)$ is the space of polynomials of degree at most equal to $r$ in each variable on $E$. Let us introduce the standard unit cube $\hat{K}=[0,1]^{3} . \forall K \in \mathscr{T}_{h}, F_{K}: \hat{K} \rightarrow K$ denotes the trilinear mapping which associates the vertices of each element. $\left(\hat{x}_{1}, \hat{x}_{2}, \hat{x}_{3}\right)$ are the coordinates on the reference element and $\left(x_{1}, x_{2}, x_{3}\right)$ the coordinates on the elements of the mesh. $D F_{K}$ and $J_{K}$ are the Jacobian matrix and its determinant associated with the map $F_{K}$.

We use the discontinuous finite element space:

$$
U_{h}=\left\{\mathbf{v}_{h} \in \mathbf{L}^{2}(\Omega): \forall K \in \mathscr{T}_{h}, D F_{K}^{*} \mathbf{v}_{h \mid K} o F_{K} \in\left[Q_{r}(\hat{K})\right]^{3}\right\}
$$

where $r \in \mathbb{N}$. 
In (2.7), the Jacobian matrix is the essential ingredient to build a conform Hingcurl approximation [21]. In our case, it allows us to reduce the storage of the stiffness and the jump matrices [34. We do not detail this point here because the aim of this paper is only the study of the convergence of this approximation. For more details on this point, we can see [34] or 4].

The first step to define the basis functions of $U_{h}$ is to construct a vector valued polynomial basis of $\left[Q_{r}\right]^{3}, \forall K \in \mathscr{T}_{h}$. We denote by $\left(\hat{\xi}_{l}, \hat{\omega}_{l}\right)_{l=1}^{r+1}$ the Gauss quadrature rule on $[0,1]$ where $\left(\hat{\xi}_{l}\right)_{l=1}^{r+1}$ are the quadrature points and $\left(\hat{\omega}_{l}\right)_{l=1}^{r+1}$ are the associated quadrature weights. The quadrature points and weights of the corresponding rules on $\hat{K}$ are the cartesian product of $1 \mathrm{D}$ points $\left\{\hat{\xi}_{l, m, n}=\left(\hat{\xi}_{l}, \hat{\xi}_{m}, \hat{\xi}_{n}\right): \forall 1 \leq l, m, n \leq\right.$ $r+1\}$ and the set $\left\{\hat{\omega}_{l, m, n}=\hat{\omega}_{l} \hat{\omega}_{m} \hat{\omega}_{n}: \forall 1 \leq l, m, n \leq r+1\right\}$ respectively. Let $\left(\hat{\varphi}_{l}\right)_{l=1}^{r+1}$ be the set of Lagrange polynomials associated with the set of points $\left(\hat{\xi}_{l}\right)_{l=1}^{r+1}$.

We have $\hat{\varphi}_{l}\left(\hat{\xi}_{j}\right)=\delta_{l, j}$ and $\left(\hat{\varphi}_{l}\right)_{l=1}^{r+1}$ is a set of basis functions of $P_{r}([0,1])=$ $Q_{r}([0,1])$.

Now, we define the basis functions of $\left[Q_{r}(\hat{K})\right]^{3}$ in the following way:

$$
\boldsymbol{\varphi}_{l, m, n}^{i}\left(\hat{x}_{1}, \hat{x}_{2}, \hat{x}_{3}\right)=\hat{\varphi}_{l}\left(\hat{x}_{1}\right) \hat{\varphi}_{m}\left(\hat{x}_{2}\right) \hat{\varphi}_{n}\left(\hat{x}_{3}\right) \mathbf{e}_{i}
$$

where $i=1,2$ or 3 and $\left(\mathbf{e}_{i}\right)_{i=1,2,3}$ is the canonical basis of $\mathbb{R}^{3}$.

We have $\varphi_{l, m, n}^{i}\left(\hat{\xi}_{l^{\prime}, m^{\prime}, n^{\prime}}\right)=\delta_{l, l^{\prime}} \delta_{m, m^{\prime}} \delta_{l, l^{\prime}} \vec{e}_{s}$. The choice of the basis functions at the quadrature points allows us to mass-lump the mass matrix [4.

Let $\mathscr{B}_{h}$ be a set of basis functions of $U_{h}$. We define an element $\boldsymbol{\psi}_{h} \in \mathscr{B}_{h}$ in the following way: $\boldsymbol{\psi}_{h} \in \mathscr{B}_{h} \Leftrightarrow \operatorname{supp}\left(\boldsymbol{\psi}_{h}\right)=K \in \mathscr{T}_{h}, \exists \varphi_{l, m, n}^{i}$ such that

$$
\boldsymbol{\psi}_{h} o F_{K}=D F_{K}^{*-1} \varphi_{l, m, n}^{i} .
$$

Let $\mathbf{v}_{h} \in U_{h}$. So, we have the decomposition:

$$
\forall K \in \mathscr{T}_{h}, \mathbf{v}_{h \mid K} o F_{K}=\sum_{i=1}^{3} \sum_{l, m, n=1}^{r+1} v_{K, l, m, n}^{i} D F_{K}^{*-1} \varphi_{l, m, n}^{i}
$$

where $v_{K, l, m, n}^{i}$ are the degrees of freedom of $\mathbf{v}_{h}$.

Finally, we obtain the following semi-discrete discontinuous Galerkin problem: find $\left(\mathbf{E}_{h}(\cdot, t), \mathbf{H}_{h}(\cdot, t)\right) \in U_{h} \times U_{h}$ such that, $\forall K \in \mathscr{T}_{h}$ and $\forall \phi_{h 1}, \phi_{h 2} \in \mathscr{B}_{h}$,

$$
\left\{\begin{array}{c}
\frac{d}{d t} \int_{K}^{G} \stackrel{\underline{\varepsilon}}{=} \mathbf{E}_{h K} \cdot \phi_{h 1 K} d x-\int_{K}^{G} \nabla \times \mathbf{H}_{h K} \cdot \phi_{h 1 K} d x \\
+\int_{K}^{G} \stackrel{\sigma \mathbf{E}_{h K} \cdot \phi_{h 1 K} d x+\int_{K}^{G} \mathbf{J}_{s} \cdot \phi_{h 1 K} d x}{=} \int_{\partial K}^{G} \beta \llbracket \mathbf{H}_{h} \times \mathbf{n}_{K} \rrbracket_{\partial K}^{K} \cdot \phi_{h 1 K} d \sigma, \\
\frac{d}{d t} \int_{K}^{G} \stackrel{\mu}{=} \mathbf{H}_{h K} \cdot \phi_{h 2 K} d x+\int_{K}^{G} \nabla \times \mathbf{E}_{h K} \cdot \phi_{h 2 K} d x \\
=\int_{\partial K}^{G} \gamma \llbracket \mathbf{E}_{h} \times \mathbf{n}_{K} \rrbracket_{\partial K}^{K} \cdot \phi_{h 2 K} d \sigma
\end{array}\right.
$$

where $\int_{K}^{G}$ and $\int_{\partial K}^{G}$ denote the integrals computed with the quadrature rule $G$ after a change of variables on the unit cube $\hat{K}$. 
Remark 2.1. Recall that the orders of the Gauss quadrature rule is $2 r+1$, i.e. exact for $\left[Q_{r}(\hat{K})\right]^{3}$.

\section{Study of A Projector on the APproximate spaCE}

In this part, we choose a projector on $U_{h}$ and we carry out its $h p$-convergence analysis. In particular, we prove some error estimates for this projector on a hexahedral mesh.

3.1. Definitions and properties of meshes. We assume that all hexahedrons $K$ are convex in order to ensure the existence of the diffeomorphism $F_{K} \in\left[Q_{1}(\hat{K})\right]^{3}$. Now let us give some definitions and properties on the quadrilateral finite elements (for more details see [1, 2]) and on the transformation $F_{K}$ : To characterize an element $K \in \mathscr{T}_{h}$, we define:

$$
\begin{gathered}
h_{K}=\text { diameter of } \mathrm{K}, \\
\sigma_{K}=\frac{h_{K}}{\rho_{K}}=\text { regularity parameter }
\end{gathered}
$$

where $\rho_{K}=\left\|J_{F_{K}^{-1}}\right\|_{\infty, K}^{\frac{1}{3}}$ with $J_{F_{K}^{-1}}$ as the determinant of the Jacobian matrix of $F_{K}^{-1}$.

Remark 3.1. In two dimensions, we can give a geometric characterization of $\rho_{K}$ (see [33]). Indeed, in this case, $\rho_{K}$ is the minimum of the diameters of the inscribed circles in the four triangles being able to be built with the nodes of the quadrangle $K$.

We note that

$$
\begin{aligned}
\left|F_{K}\right|_{m, \infty, \hat{K}} & =\sup _{\hat{\mathbf{x}} \in \hat{K}}\left\|D^{m} F_{K}(\hat{\mathbf{x}})\right\|_{\mathscr{L}_{m}\left(\mathbb{R}^{3}, \mathbb{R}^{3}\right)}, \\
\left|F_{K}^{-1}\right|_{m, \infty, K} & =\sup _{\mathbf{x} \in K}\left\|D^{m} F_{K}^{-1}(\mathbf{x})\right\|_{\mathscr{L}_{m}\left(\mathbb{R}^{3}, \mathbb{R}^{3}\right)}
\end{aligned}
$$

where $\mathscr{L}_{m}\left(\mathbb{R}^{3}, \mathbb{R}^{3}\right)$ is the set of the $m$-linear applications of $\mathbb{R}^{3}$ in $\mathbb{R}^{3}, D^{m} F_{K}(\hat{\mathbf{x}})$ and $D^{m} F_{K}^{-1}(\mathbf{x})$ are respectively the $m$ th derivatives of $F_{K}$ and $F_{K}^{-1}$ at the points $\hat{\mathbf{x}}$ and $\mathbf{x}$. We will use the following estimates given in [2]:

$$
\begin{aligned}
& \left|F_{K}\right|_{1, \infty, \hat{K}} \leq C h_{K},\left\|J_{K}\right\|_{\infty, \hat{K}} \leq C h_{K}^{3}, \\
& \left|F_{K}^{-1}\right|_{1, \infty, K} \leq C \frac{h_{K}^{2}}{\rho_{K}^{3}},\left\|J_{F_{K}^{-1}}\right\|_{\infty, K}=\rho_{K}^{-3}, \\
& \left|F_{K}\right|_{2, \infty, \hat{K}} \leq C h_{K},\left|F_{K}\right|_{2, \infty, \hat{K}} \leq C h_{K}^{2} \text { if } K \text { is almost a parallelepiped }
\end{aligned}
$$

where $C>0$ is independent of $K$ and $r$.

Remark 3.2. By the expression "almost a parallelepiped", one wants to say a small deformation of a parallelepipedic cell. In this case, the second derivatives of $F_{K}$ are zero. 
Remark 3.3. We have by definition

$$
\begin{aligned}
& D\left(F_{K}^{-1}\right)(\mathbf{x})=\left(D F_{K}\left(F^{-1}(\mathbf{x})\right)\right)^{-1}, \\
& J_{F_{K}^{-1}} o F_{K}=\frac{1}{J_{K}}
\end{aligned}
$$

where $D\left(F_{K}^{-1}\right)$ is the Jacobian matrix of $F_{K}^{-1}$.

Using the properties (3.3), it is easy to deduce the following proposition:

Proposition 1. We have the following estimates: $\forall \hat{x} \in \hat{K}$,

$$
\begin{gathered}
\lambda\left(\left(D F_{K} D F_{K}^{*}\right)(\hat{x})\right) \leq C h_{K}^{2}, \\
\lambda\left(\left(D F_{K}^{-1} D F_{K}^{*-1}\right)(\hat{x})\right) \leq C \frac{h_{K}^{4}}{\rho_{K}^{6}}
\end{gathered}
$$

where $\lambda(A)$ belongs to the spectrum of $A$ and $C>0$ is independent of $K$ and $r$.

Proof. Let $\hat{x} \in \hat{K}$. As $\left(D F_{K} D F_{K}^{*}\right)(\hat{x})$ and $\left(D F_{K}^{-1} D F_{K}^{*-1}\right)(\hat{x})$ are symmetrical matrices, we can write:

$$
\begin{gathered}
\rho\left(\left(D F_{K} D F_{K}^{*}\right)(\hat{x})\right)=\sup _{\mathbf{v} \in \mathbb{R}^{* 3}} \frac{\left\|\left(D F_{K} D F_{K}^{*}\right)(\hat{x}) \mathbf{v}\right\|}{\|\mathbf{v}\|}=\left\|\left(D F_{K} D F_{K}^{*}\right)(\hat{x})\right\| \\
\leq\left\|\left(D F_{K}\right)(\hat{x})\right\|\left\|\left(D F_{K}^{*}\right)(\hat{x})\right\| \leq\left|F_{K}\right|_{1, \infty, \hat{K}}^{2} ;
\end{gathered}
$$

$\rho(A)$ is the spectral radius of $A$. Using (3.3), we immediately obtain the first inequality of (3.5). A similar reasoning allows us to prove the second estimate of (3.5).

Finally, we define the regularity of a mesh:

Definition 3.4. A family $\mathscr{T}_{h}$ of triangulation of $\Omega$ is known as regular when $h$ tends toward 0 , if there exists a number $\sigma>0$, independent of $h$, such that:

$$
\sigma_{K} \leq \sigma, \forall K \in \mathscr{T}_{h}
$$

3.2. Choice of a projector. When deriving error estimates, an important point is the choice of a "good" projector on the approximate space used for discretization. Indeed, the use of an inappropriate projector can lead to sub-optimal estimates which give any interesting information about the numerical scheme. This part aims at justifying our choice.

For our DG scheme, the first idea is to use an $L^{2}$ projector. In particular, one can use the projector defined in the following way:

First, we can split the approximate space $U_{h}$ in the following way:

$$
U_{h}=\bigoplus_{K \in \mathscr{T}_{h}} U_{K}
$$

where $U_{K}=\left\{\mathbf{v} \in \mathbf{L}^{2}(K): D F_{K}^{*} v o F_{K} \in\left[Q_{r}(\hat{K})\right]^{3}\right\}$.

Then, in the first step, we define the $L^{2}$ projector $\hat{\pi}_{r}^{0}$ on $\left[Q_{r}(\hat{K})\right]^{3}$ :

Definition 3.5 (Projector $L^{2}$ ). Let $\hat{\mathbf{v}} \in \mathbf{L}^{2}(\hat{K})$ and $r \geq 0$. We define the projector $L^{2}, \hat{\pi}_{r}^{0} \hat{\mathbf{v}}$, of $\hat{\mathbf{v}}$ on $\left[Q_{r}(\hat{K})\right]^{3}$ by $: \forall \hat{\varphi} \in\left[Q_{r}(\hat{K})\right]^{3}$, we have

$$
\int_{\hat{K}} \hat{\pi}_{r}^{0} \hat{\mathbf{v}} \cdot \hat{\varphi} d \hat{\mathbf{x}}=\int_{\hat{K}} \hat{\mathbf{v}} \cdot \hat{\varphi} d \hat{\mathbf{x}}
$$


In the second step, we come back to $U_{K}$ by defining the projector $\pi_{K}^{0}$.

Definition 3.6 (Projector on $U_{K}$ ). Let $\mathbf{v} \in \mathbf{L}^{2}(K)$. We define the projection $\pi_{K}^{0} \mathbf{v}$ of $\mathbf{v}$ on $U_{K}$ by

$$
\left(\pi_{K}^{0} \mathbf{v}\right) o F_{K}=D F_{K}^{*-1} \hat{\pi}_{r}^{0} \hat{\mathbf{v}}
$$

where $\hat{\mathbf{v}}=D F_{K}^{*} \mathbf{v} \circ F_{K}$.

Finally we define the projection operator on $U_{h}$.

Definition 3.7 (Projector on $U_{h}$ ). Let $\mathbf{v} \in \mathbf{L}^{2}(\Omega)$. We define the projection $\pi_{h}^{0} \mathbf{v}$ of $\mathbf{v}$ on $U_{h}$ by: For $K \in \mathscr{T}_{h}$,

$$
\left(\pi_{h}^{0} \mathbf{v}\right)_{\mid K}=\pi_{K}^{0} \mathbf{v}_{\mid K}
$$

When examining the DG scheme in more detail, one sees that it is necessary to know error estimates of the first order derivatives of the projector used (because of the presence of the rational terms). So, an $H^{1}$ type projector on $U_{h}$ can be a possibility for this study. In particular, we have considered the projector defined as:

First, we define the $H^{1}$ projector $\hat{\pi}_{r}^{1}$ on $\left[Q_{r}(\hat{K})\right]^{3}$.

Definition 3.8 (Projector $H^{1}$ ). Let $\hat{\mathbf{v}} \in \mathbf{H}^{1}(\hat{K})$ and $r \geq 0$. We define the $H^{1}$ projection, $\hat{\pi}_{r}^{1} \hat{\mathbf{v}}$, of $\hat{\mathbf{v}}$ on $\left[Q_{r}(\hat{K})\right]^{3}$ by $\forall \hat{\varphi} \in\left[Q_{r}(\hat{K})\right]^{3}$, we have

$$
\int_{\hat{K}}\left(\hat{\pi}_{r}^{1} \hat{\mathbf{v}}-\hat{\mathbf{v}}\right) \cdot \hat{\boldsymbol{\varphi}} d \hat{\mathbf{x}}+\sum_{k=1}^{3} \int_{\hat{K}} \frac{\partial}{\partial \hat{x}_{k}}\left(\hat{\pi}_{r}^{1} \hat{\mathbf{v}}-\hat{\mathbf{v}}\right) \cdot \frac{\partial}{\partial \hat{x}_{k}} \hat{\boldsymbol{\varphi}} d \hat{\mathbf{x}}=0 .
$$

Remark 3.9. In (3.12), $\frac{\partial \mathbf{w}}{\partial \hat{x}_{k}}$ means $\left(\frac{\partial w_{1}}{\partial \hat{x}_{k}}, \frac{\partial w_{2}}{\partial \hat{x}_{k}}, \frac{\partial w_{3}}{\partial \hat{x}_{k}}\right)^{*}$.

Then, we come back to $U_{K}$. Let $K \in \mathscr{T}_{h}$ and $\mathbf{v} \in \mathbf{H}^{s}(K)$ with $s \geq 1$. We define the projector $\pi_{K}^{1}$ on $U_{K}$ by

$$
\left(\pi_{K}^{1} \mathbf{v}\right) o F_{K}=D F_{K}^{*-1}\left(\hat{\pi}_{r}^{1} \hat{\mathbf{v}}\right)
$$

where $\hat{\mathbf{v}}=D F_{K}^{*}\left(\mathbf{v} o F_{K}\right)$.

Finally we define the projection operator on $U_{h}$.

Definition 3.10 (Projector on $U_{h}$ ). Let $\mathbf{v} \in \mathbf{L}^{2}(\Omega)$. We define the projection $\pi_{h}^{1} \mathbf{v}$ of $\mathbf{v}$ on $U_{h}$ by: For $K \in \mathscr{T}_{h}$,

$$
\left(\pi_{h}^{1} \mathbf{v}\right)_{\mid K}=\pi_{K}^{1} \mathbf{v}_{\mid K}
$$

We must be able to discriminate against these two projectors. The following subsection (" $h p$-projection errors") shows that the study identically applies to the two projectors and consequently gives the same interpolation error estimates. Moreover, section 4 shows that the two projectors lead to the same $h$ convergence rate. However, the study of the spectral or of the $h p$ convergence shows that these projectors do not give the same result:

Using theorem 57 of 31 as well as a tensorisation argument (i.e. $\hat{\pi}_{r}^{0}=\hat{\pi}_{r, \hat{x}_{3}}^{0} o \hat{\pi}_{r, \hat{x}_{2}}^{0}$ $\left.o \hat{\pi}_{r, \hat{x}_{1}}^{0}\right)$, we obtain the projection errors for $\hat{\pi}_{r}^{0}$ :

Theorem 3.11. $\forall \hat{\mathbf{u}} \in \mathbf{H}^{p}(\hat{K})$, it exists a constant $C$ such that

$$
\left\|\hat{\mathbf{u}}-\hat{\pi}_{r}^{0} \hat{\mathbf{u}}\right\|_{q, \hat{K}} \leq C r^{\sigma(p, q)}\|\hat{\mathbf{u}}\|_{p, \hat{K}}
$$


where

$$
\sigma(p, q)=\left\{\begin{array}{l}
\frac{3}{2} q-p, 0 \leq q \leq 1 \\
2 q-p-\frac{1}{2} q, q \geq 1
\end{array}\right.
$$

and $0 \leq q \leq p$.

As already mentioned, we need the $H^{1}$ projection error to estimate the error of the GD scheme. The previous theorem gives us:

$$
\left\|\hat{\mathbf{u}}-\hat{\pi}_{r}^{0} \hat{\mathbf{u}}\right\|_{1, \hat{K}} \leq C r^{\frac{3}{2}-p}\|\hat{\mathbf{u}}\|_{p, \hat{K}} .
$$

(3.17) shows that we do not have the optimality for the $H^{1}$ norm.

However, for $\hat{\pi}_{r}^{1}$, we can find in [30] the following estimate: $\forall t, s \in \mathbb{R}$ verifying $0 \leq t \leq 1 \leq s$, then for $\hat{\mathbf{v}} \in \mathbf{H}^{s}(\hat{K})$, there exists a constant $C>0$ independent of $r$ such that:

$$
\left\|\hat{\mathbf{v}}-\hat{\pi}_{r}^{1} \hat{\mathbf{v}}\right\|_{t, \hat{K}} \leq C r^{t-s}\|\hat{\mathbf{v}}\|_{s, \hat{K}}
$$

In particular, we will use the two estimates: $(t=0,1$ in (3.18) $)$.

Proposition 2. For $\hat{\mathbf{v}} \in \mathbf{H}^{s}(\hat{K}), s \geq 1$,

$$
\begin{gathered}
\left\|\hat{\mathbf{v}}-\hat{\pi}_{r}^{1} \hat{\mathbf{v}}\right\|_{0, \hat{K}} \leq \frac{C}{r^{s}}\|\hat{\mathbf{v}}\|_{s, \hat{K}}, \\
\left\|\hat{\mathbf{v}}-\hat{\pi}_{r}^{1} \hat{\mathbf{v}}\right\|_{1, \hat{K}} \leq \frac{C}{r^{s-1}}\|\hat{\mathbf{v}}\|_{s, \hat{K}}
\end{gathered}
$$

where $C>0$ is a constant independent of $r$.

In this case, we obtain the optimal projection errors $\left(1 / r^{s}\right.$ and $1 / r^{s-1}$ for the $L^{2}$ and the $H^{1}$ norms respectively). In conclusion, we have decided to use the projector $\pi_{h}^{1}$ to analyze the convergence properties of the DG scheme in the $h p$-version.

3.3. $h p$-projection errors. To study the projection error introduced by $\hat{\pi}_{r}^{1}$, we use the bracket semi-norm: Let $u \in W^{m, p}(\hat{K})$,

$$
[u]_{m, p, \hat{K}}^{2}=\sum_{i=1}^{3}\left\|\frac{\partial^{m} u}{\partial \hat{x}_{i}^{m}}\right\|_{p, \hat{K}}^{2} .
$$

and the Bramble-Hilbert lemma adapted to $Q_{r}$ (see [33, [1], 2]):

Lemma 3.12 (Bramble-Hilbert). Let $p, q$ be two numbers such that $1 \leq p, q \leq \infty$ and let $r, m$ be two integers such that $r \geq 0$ and $m \leq r+1$,

$$
W^{r+1, p}(\hat{K}) \hookrightarrow W^{m, q}(\hat{K}) .
$$

Let $\Pi \in \mathscr{L}\left(W^{r+1, p}(\hat{K}) ; W^{m, q}(\hat{K})\right)$ be an operator which verifies

$$
\forall p \in Q_{r}, \Pi p=p .
$$

Then there exists $C$ dependent on $\hat{K}$ and $r$ such that

$$
\forall v \in W^{r+1, p}(\hat{K}),|v-\Pi v|_{m, q, \hat{K}} \leq C[v]_{r+1, p, \hat{K}} .
$$


In (3.23),$|\cdot|_{m, q, \hat{K}}$ is the semi-norm defined by: Let $v \in W^{m, q}(\hat{K})$,

$$
|v|_{m, q, \hat{K}}=\left(\sum_{|\alpha|=m} \int_{\hat{K}}\left|\frac{\partial^{|\alpha|}}{\partial \hat{\mathbf{x}}^{\alpha}} v\right|^{q} d \hat{\mathbf{x}}\right)^{\frac{1}{q}} .
$$

The Bramble-Hilbert lemma applied to the operator $\hat{\pi}_{r}^{1}$, immediately leads to:

Proposition 3. For $r \geq 0$ and $m \leq r+1$, there exists $C$ dependent on $\hat{K}$ and $r$ such that:

$$
\forall \hat{\mathbf{v}} \in \mathbf{H}^{r+1}(\hat{K}),\left|\hat{\mathbf{v}}-\hat{\pi}_{r}^{1} \hat{\mathbf{v}}\right|_{m, \hat{K}} \leq C[\mathbf{v}]_{r+1, \hat{K}} .
$$

In order to derive the $h p$-projection error estimates for $\pi_{h}^{1}$, we must specify the exact $r$-dependence of the constant $C$ of (3.24). To do so, we come back to the proof of the Bramble-Hilbert lemma but directly considering $\pi_{h}^{1}$. The first step, to prove this type of result, is to write [1]: $\forall \hat{\mathbf{v}} \in \mathbf{H}^{r+1}(\hat{K})$,

$$
\begin{aligned}
\left|\hat{\mathbf{v}}-\hat{\pi}_{r}^{1} \hat{\mathbf{v}}\right|_{m, \hat{K}} & \leq\left\|I-\hat{\pi}_{r}^{1}\right\|_{\mathscr{L}\left(\mathbf{H}^{r+1}(\hat{K}), H^{m}(\hat{K})\right)} \inf _{\hat{p} \in\left[Q_{r}(\hat{K})\right]^{3}}\|\hat{\mathbf{v}}+p\|_{r+1, \hat{K}} \\
& \leq C_{1}\left\|I-\hat{\pi}_{r}^{1}\right\|_{\mathscr{L}\left(\mathbf{H}^{r+1}(\hat{K}), H^{m}(\hat{K})\right)}[\mathbf{v}]_{r+1, \hat{K}}
\end{aligned}
$$

where $C_{1}$ is independent of $r$.

By using (3.18), (3.25) we immediately get:

$$
\left|\hat{\mathbf{v}}-\hat{\pi}_{r}^{1} \hat{\mathbf{v}}\right|_{m, \hat{K}} \leq \frac{C_{2}(\hat{K})}{r^{r+1-m}}[\mathbf{v}]_{r+1, \hat{K}}, 0 \leq m \leq r+1 .
$$

In order to determine the projector errors, we will need the following estimate :

Lemma 3.13. Let $K \in \mathscr{T}_{h}$ and $v \in W^{m, p}(K)$. We have the estimate:

$$
\left[v \circ F_{K}\right]_{m, p, \hat{K}} \leq C \frac{h_{K}^{m}}{\rho_{K}^{\frac{3}{p}}}|v|_{m, p, K} .
$$

If $\mathscr{T}_{h}$ belongs to a regular family of triangulation, we give:

$$
\left[v \circ F_{K}\right]_{m, p, \hat{K}} \leq C \sigma^{\frac{3}{p}} h_{K}^{m-\frac{3}{p}}|v|_{m, p, K}
$$

where $C>0$ independent of $K$ and $r$.

Proof. Note $F_{K}=\left(F_{K}^{1}, F_{K}^{2}, F_{K}^{3}\right)$. To prove this lemma, we use the property:

$$
\partial_{\hat{x}_{k}^{2}}^{2} F_{K}^{i}=0 \text { for } i=1,2,3,
$$

because $F_{K}^{i} \in Q_{1}(\hat{K})$.

Let $\mathbf{v} \in \mathbf{H}^{r+1}(K), r \geq 0$.

Lemma 3.14. There exists $C$ independent of $K$ and $r$ such that:

$$
\begin{gathered}
\left\|\mathbf{v}-\pi_{K}^{1} \mathbf{v}\right\|_{0, K} \leq C \frac{h_{K}^{\frac{1}{2}}}{r^{r+1}}[\hat{\mathbf{v}}]_{r+1, \hat{K}} \\
\left|\mathbf{v}-\pi_{K}^{1} \mathbf{v}\right|_{1, K} \leq \frac{C}{h_{K}^{\frac{1}{2}} r^{r}}[\hat{\mathbf{v}}]_{r+1, \hat{K}}
\end{gathered}
$$


Proof. We prove only the second inequality. It suffices to use the same process to obtain the first. Write $\mathbf{w}=\mathbf{v}-\pi_{K}^{1} \mathbf{v}=\left(w_{1}, w_{2}, w_{3}\right)^{*}$ (* reads for the transposition operator). We have

$$
|\mathbf{w}|_{1, K}^{2}=\sum_{i=1}^{3} \sum_{l=1}^{3} \int_{\hat{K}}\left|J_{K} \|\left(\partial_{x_{l}} w_{i}\right) o F_{K}\right|^{2} d \hat{\mathbf{x}}
$$

where the notation $\partial_{x_{l}}$ means $\frac{\partial}{\partial x_{l}}$. By definition, we have $\mathbf{w}=D F_{K}^{*-1} o F_{K}^{-1} \hat{\mathbf{w}} o F_{K}^{-1}$ where $\hat{\mathbf{w}}=\hat{\mathbf{v}}-\hat{\pi}_{r}^{1} \hat{\mathbf{v}}$ and $D F_{K}^{*}$ writes:

$$
D F_{K}^{*}=\left(\begin{array}{ccc}
\partial_{\hat{x}_{1}} x_{1} & \partial_{\hat{x}_{1}} x_{2} & \partial_{\hat{x}_{1}} x_{3} \\
\partial_{\hat{x}_{2}} x_{1} & \partial_{\hat{x}_{2}} x_{2} & \partial_{\hat{x}_{2}} x_{3} \\
\partial_{\hat{x}_{3}} x_{1} & \partial_{\hat{x}_{3}} x_{2} & \partial_{\hat{x}_{3}} x_{3}
\end{array}\right)
$$

where $x_{i}=F_{K}^{i}(\hat{\mathbf{x}})$ for $i=1,2,3$.

Inverting this matrix with the help of the co-factors formula, we obtain:

$$
\begin{gathered}
D F_{K}^{*-1}=\frac{1}{J_{K}} \\
\left(\begin{array}{ccc}
\partial_{\hat{x_{2}}} x_{2} \partial_{\hat{x_{3}}} x_{3}-\partial_{\hat{x_{2}}} x_{3} \partial_{\hat{x_{3}}} x_{2} & -\partial_{\hat{x_{2}}} x_{1} \partial_{\hat{x_{3}}} x_{3}+\partial_{\hat{x_{2}}} x_{3} \partial_{\hat{x_{3}}} x_{1} & \partial_{\hat{x_{2}}} x_{1} \partial_{\hat{x_{3}}} x_{2}-\partial_{\hat{x_{2}}} x_{2} \partial_{\hat{x_{3}}} x_{1} \\
-\partial_{\hat{x_{1}}} x_{2} \partial_{\hat{x_{3}}} x_{3}+\partial_{\hat{x_{1}}} x_{3} \partial_{\hat{x_{3}}} x_{2} & \partial_{\hat{x_{1}}} x_{1} \partial_{\hat{x_{3}}} x_{3}-\partial_{\hat{x_{1}}} x_{3} \partial_{\hat{x_{3}}} x_{1} & -\partial_{\hat{x_{1}}} x_{1} \partial_{\hat{x_{3}}} x_{2}+\partial_{\hat{x_{1}}} x_{2} \partial_{\hat{x_{3}}} x_{1} \\
\partial_{\hat{x_{1}}} x_{2} \partial_{\hat{x_{2}}} x_{3}-\partial_{\hat{x_{1}}} x_{3} \partial_{\hat{x_{2}}} x_{2} & -\partial_{\hat{x_{1}}} x_{1} \partial_{\hat{x_{2}}} x_{3}+\partial_{\hat{x_{1}}} x_{3} \partial_{\hat{x_{2}}} x_{1} & \partial_{\hat{x_{1}}} x_{1} \partial_{\hat{x_{2}}} x_{2}-\partial_{\hat{x_{1}}} x_{2} \partial_{\hat{x_{2}}} x_{1}
\end{array}\right) .
\end{gathered}
$$

Note that $D F_{K}^{*-1}=\frac{1}{J_{K}}\left(m_{i, j}\right)_{i, j=1, \cdots, 3}$, so we have $w_{i}=\sum_{j=1}^{3} \frac{m_{i, j} o F_{K}^{-1}}{J_{K} o F_{K}^{-1}} \hat{w}_{j} o F_{K}^{-1}$.

Now, we derive the last expression with respect to $x_{l}$ :

$$
\begin{gathered}
\partial_{x_{l}} w_{i}=\sum_{j=1}^{3}\left[\frac{\partial_{x_{l}}\left(m_{i, j} o F_{K}^{-1}\right) J_{K} o F_{K}^{-1}-m_{i, j} o F_{K}^{-1} \partial_{x_{l}}\left(J_{K} o F_{K}^{-1}\right)}{\left(J_{K} o F_{K}^{-1}\right)^{2}} \hat{w}_{j} o F_{K}^{-1}\right. \\
\left.+\frac{m_{i, j} o F_{K}^{-1}}{J_{K} o F_{K}^{-1}} \partial_{x_{l}}\left(\hat{w}_{j} o F_{K}^{-1}\right)\right] \\
=\sum_{j=1}^{3}\left[\sum_{k=1}^{3} \frac{\left(\partial_{\hat{x}_{k}} m_{i, j}\right) o F_{K}^{-1} \partial_{x_{l}} \hat{x}_{k} J_{K} o F_{K}^{-1}-m_{i, j} o F_{K}^{-1}\left(\partial_{\hat{x}_{k}} J_{K}\right) o F_{K}^{-1} \partial_{x_{l}} \hat{x}_{k}}{\left(J_{K} o F_{K}^{-1}\right)^{2}} \hat{w}_{j} o F_{K}^{-1}\right. \\
\left.+\frac{m_{i, j} o F_{K}^{-1}}{J_{K} o F_{K}^{-1}}\left(\partial_{\hat{x}_{k}} \hat{w}_{j}\right) o F_{K}^{-1} \partial_{x_{l}} \hat{x}_{k}\right]
\end{gathered}
$$

Note that

$$
\begin{gathered}
T_{i, j}^{k, l}=\frac{\left(\partial_{\hat{x}_{k}} m_{i, j}\right) \partial_{x_{l}} \hat{x}_{k} o F_{K} J_{K}-m_{i, j}\left(\partial_{\hat{x}_{k}} J_{K}\right) \partial_{x_{l}} \hat{x}_{k} o F_{K}}{\left(J_{K}\right)^{2}} \\
\tilde{T}_{i, j}^{k, l}=\frac{m_{i, j}}{J_{K}} \partial_{x_{l}} \hat{x}_{k} o F_{K}
\end{gathered}
$$


so we can write:

$$
\left(\partial_{x_{l}} w_{i}\right) o F_{K}=\sum_{j, k=1}^{3}\left[T_{i, j}^{k, l} \hat{w}^{j}+\tilde{T}_{i, j}^{k, l} \partial_{\hat{x}_{k}} \hat{w}^{j}\right] .
$$

The mesh regularity leads to:

$$
\begin{aligned}
& \left|T_{i, j}^{k, l}\right| \leq \frac{C}{h_{K}^{2}}, \\
& \left|\tilde{T}_{i, j}^{k, l}\right| \leq \frac{C}{h_{K}^{2}}
\end{aligned}
$$

where $C>0$ independent of $K$ and $r$. Indeed, the definition of $m_{i, j}$ gives us $\left|m_{i, j}\right| \leq C h_{K}^{2}$ and $\left|\partial_{\hat{x}_{k}} m_{i, j}\right| \leq C h_{K}^{2}$ (keep in mind that $x_{i}=F_{K}^{i}(\mathbf{x})$ for $i \in \llbracket 1,3 \rrbracket$ ). Moreover, the estimates (3.3) imply $\left|\partial_{x_{l}} \hat{x}_{k} o F_{K}\right| \leq C / h_{K},\left|J_{K}\right| \leq C h_{K}^{3},\left|\partial_{\hat{x}_{k}} J_{K}\right| \leq$ $C h_{K}^{3}$ and $\left|J_{K}\right| \geq C^{\prime} h_{K}^{3}$. That allows us to obtain:

$$
\left|\left(\partial_{x_{l}} w_{i}\right) o F_{K}\right|^{2} \leq \frac{C}{h_{K}^{4}} \sum_{j, k=1}^{3}\left[\left|\hat{w}_{j}\right|^{2}+\left|\partial_{\hat{x}_{k}} \hat{w}_{j}\right|^{2}\right] .
$$

Return to our semi-norm: Using (3.37), (3.31) leads to

$$
\begin{gathered}
|\mathbf{w}|_{1, K}^{2} \leq C \frac{\left\|J_{K}\right\|_{\infty, \hat{K}}}{h_{K}^{4}} \sum_{i=1}^{3} \sum_{l=1}^{3} \sum_{j, k=1}^{3} \int_{\hat{K}}\left[\left|\hat{w}_{j}\right|^{2}+\left|\partial_{\hat{x}_{k}} \hat{w}_{j}\right|^{2}\right] d \hat{x} \\
\leq \frac{C}{h_{K}}\|\hat{\mathbf{w}}\|_{1, \hat{K}}^{2} .
\end{gathered}
$$

Finally (3.26) gives the lemma.

The following step is to increase $[\hat{\mathbf{v}}]_{m, \hat{K}}$ by a power of $h_{K}$ and $\|\mathbf{v}\|_{m, K}$.

Lemma 3.15. Let $\mathbf{v} \in \mathbf{H}^{m}(K)$. We have the following estimate:

$$
[\hat{\mathbf{v}}]_{m, \hat{K}} \leq C \sum_{l=0}^{1}\left|F_{K}\right|_{l+1, \infty, \hat{K}}\left[\mathbf{v} o F_{K}\right]_{m-l, \hat{K}}
$$

where $C>0$ independent of $K$ and $r$.

Proof. We have $\hat{\mathbf{v}}=D F_{K}^{*} \mathbf{v} o F_{K}$ and $[\hat{\mathbf{v}}]_{m, \hat{K}}^{2}=\sum_{i=1}^{3} \sum_{j=1}^{3} \int_{\hat{K}}\left|\frac{\partial^{m} \hat{v}_{j}}{\partial \hat{x}_{i}^{m}}\right|^{2} d \hat{x}$. We can write $\hat{v}_{j}=\sum_{k=1}^{3} J_{j, k} v_{k} o F$ where $D F_{K}^{*}=\left(J_{j, k}\right)_{j, k=1, \cdots, 3}$. The Leibniz formula leads to:

$$
\frac{\partial^{m} \hat{v}_{j}}{\partial \hat{x}_{i}^{m}}=\sum_{k=1}^{3} \sum_{l=0}^{m}\left(\begin{array}{c}
l \\
m
\end{array}\right) \frac{\partial^{l}\left(J_{j, k}\right)}{\partial \hat{x}_{i}^{l}} \frac{\partial^{m-l}\left(v_{k} o F\right)}{\partial \hat{x}_{i}^{m-l}} .
$$


For $l \geq 2$, we have $\frac{\partial^{l}\left(J_{j, k}\right)}{\partial \hat{x}_{i}^{l}}=0$ (indeed $\left.F_{K} \in\left[Q_{1}(\hat{K})\right]^{3}\right)$. That implies:

$$
\begin{gathered}
\int_{\hat{K}}\left|\frac{\partial^{m} \hat{v}_{j}}{\partial \hat{x}_{i}^{m}}\right|^{2} d \hat{x} \leq C \sum_{k=1}^{3} \sum_{l=0}^{1}\left|F_{K}\right|_{l+1, \infty, \hat{K}}^{2} \int_{\hat{K}}\left|\frac{\partial^{m-l}\left(v_{k} o F\right)}{\partial \hat{x}_{i}^{m-l}}\right|^{2} d \hat{x} \\
\leq C \sum_{k=1}^{3} \sum_{l=0}^{1}\left|F_{K}\right|_{l+1, \infty, \hat{K}}^{2}\left[v_{k} o F_{K}\right]_{m-l, \hat{K}}^{2} .
\end{gathered}
$$

So, we obtain the following result:

$$
\begin{aligned}
{[\hat{\mathbf{v}}]_{m, \hat{K}}^{2} } & \leq C \sum_{i=1}^{3} \sum_{j=1}^{3} \sum_{k=1}^{3} \sum_{l=0}^{1}\left|F_{K}\right|_{l+1, \infty, \hat{K}}^{2}\left[v_{k} o F_{K}\right]_{m-l, \hat{K}}^{2} \\
& \leq C \sum_{l=0}^{1}\left|F_{K}\right|_{l+1, \infty, \hat{K}}^{2}\left[\mathbf{v} o F_{K}\right]_{m-l, \hat{K}}^{2} \cdot \square
\end{aligned}
$$

Finally, by grouping (3.30), (3.28) and (3.39) together, we obtain the following error estimates:

Proposition 4. Let $\mathbf{v} \in \mathbf{H}^{r+1}(K)$. Then there exists $C$ independent of the cell $K$ and $r$ such that:

$$
\begin{gathered}
\left\|\mathbf{v}-\pi_{K}^{1} \mathbf{v}\right\|_{0, K} \leq C \frac{h_{K}^{r}}{r^{r+1}}\left(|\mathbf{v}|_{r, K}+h_{K}|\mathbf{v}|_{r+1, K}\right), \\
\left|\mathbf{v}-\pi_{K}^{1} \mathbf{v}\right|_{1, K} \leq C \frac{h_{K}^{r-1}}{r^{r}}\left(|\mathbf{v}|_{r, K}+h_{K}|\mathbf{v}|_{r+1, K}\right) .
\end{gathered}
$$

Now, by using the interpolation Theorem 1.4 of [33], we extend the result to the real exponents.

Proposition 5. Let $\mathbf{v} \in \mathbf{H}^{s+1}(K)$, for $0 \leq s \leq r$ real and assume that $0<h_{K} \leq 1$. Then there exists $C$ independent of the cell $K$ and $r$ and such that:

$$
\begin{aligned}
& \left\|\mathbf{v}-\pi_{K}^{1} \mathbf{v}\right\|_{0, K} \leq C \frac{h_{K}^{s}}{r^{s+1}}\|\mathbf{v}\|_{s+1, K}, \\
& \left|\mathbf{v}-\pi_{K}^{1} \mathbf{v}\right|_{1, K} \leq C \frac{h_{K}^{s-1}}{r^{s}}\|\mathbf{v}\|_{s+1, K} .
\end{aligned}
$$

Proof. Let $r 1<r 2$ be two positive integers and $\theta \in[0,1]$. Assume that $\pi_{K}^{0} \in$ $\mathscr{L}\left(\mathbf{H}^{r 1+1}(K), H^{m}(K)\right) \cap \mathscr{L}\left(\mathbf{H}^{r 2+1}(K), H^{m}(K)\right)$ for $m=0,1$. Then we have:

$$
\begin{gathered}
\left\|I-\pi_{K}^{1}\right\|_{\mathscr{L}\left(\mathbf{H}^{\theta r 1+(1-\theta) r 2+1}(K), H^{m}(K)\right)} \\
\leq C\left\|I-\pi_{K}^{1}\right\|_{\mathscr{L}\left(\mathbf{H}^{r 1+1}(K), H^{m}(K)\right)}^{\theta}\left\|I-\pi_{K}^{1}\right\|_{\mathscr{L}\left(\mathbf{H}^{r 2+1}(K), H^{m}(K)\right)}^{1-\theta} .
\end{gathered}
$$

The inequalities (3.43) lead to:

$$
\begin{aligned}
\left\|I-\pi_{K}^{1}\right\|_{\mathscr{L}\left(\mathbf{H}^{r 1+1}(K), H^{m}(K)\right)} & \leq C \frac{h_{K}^{r 1-m}}{r^{r 1+1-m}}, \\
\left\|I-\pi_{K}^{1}\right\|_{\mathscr{L}\left(\mathbf{H}^{r 2+1}(K), H^{m}(K)\right)} & \leq C \frac{h_{K}^{r 2-m}}{r^{r 2+1-m}} .
\end{aligned}
$$


So we obtain:

$$
\left\|I-\pi_{K}^{1}\right\|_{\mathscr{L}\left(\mathbf{H}^{\theta r 1+(1-\theta) r 2+1}(K), H^{m}(K)\right)} \leq C \frac{h_{K}^{\theta r 1+(1-\theta) r 2-m}}{r^{\theta r 1+(1-\theta) r 2+1-m}} .
$$

Finally, take $r 1=0, r 2=r$ and $s=(1-\theta)$. We can write the inequality:

$$
\begin{aligned}
\left\|\mathbf{v}-\pi_{K}^{1} \mathbf{v}\right\|_{m, K} & \leq\left\|I-\pi_{K}^{1}\right\|_{\mathscr{L}\left(\mathbf{H}^{s+1}(K), H^{m}(K)\right)}\|\mathbf{v}\|_{s+1, K} \\
& \leq C \frac{h_{K}^{s-m}}{r^{s+1-m}}\|\mathbf{v}\|_{s+1, K} \cdot \square
\end{aligned}
$$

Now, if we take $\mathbf{v} \in \mathbf{H}^{s}(K)$ with $s \geq r+1$, we prove easily the error estimates:

$$
\begin{gathered}
\left\|\mathbf{v}-\pi_{K}^{1} \mathbf{v}\right\|_{0, K} \leq C \frac{h_{K}^{r}}{r^{s}}\|\mathbf{v}\|_{s, K}, \\
\left|\mathbf{v}-\pi_{K}^{1} \mathbf{v}\right|_{1, K} \leq C \frac{h_{K}^{r-1}}{r^{s-1}}\|\mathbf{v}\|_{s, K} .
\end{gathered}
$$

Finally, (3.43) and (3.45) lead to the global result: Let $\mathbf{v} \in \mathbf{H}^{s+1}(K)$ with $s \geq 0$ :

$$
\begin{gathered}
\left\|\mathbf{v}-\pi_{K}^{1} \mathbf{v}\right\|_{0, K} \leq C \frac{h_{K}^{\min (s, r)}}{r^{s+1}}\|\mathbf{v}\|_{s+1, K}, \\
\left|\mathbf{v}-\pi_{K}^{1} \mathbf{v}\right|_{1, K} \leq C \frac{h_{K}^{\min (s-1, r-1)}}{r^{s}}\|\mathbf{v}\|_{s+1, K}
\end{gathered}
$$

where $C$ is independent of the cell $K$ and $r$.

\section{A-PRiori ERROR ESTimates FOR the SPATIAL SEMI-DISCRETE APPROXIMATION}

In this part, we consider that all the integrals are computed in an exact way. Let $(\mathbf{E}, \mathbf{H})$ and $\left(\mathbf{E}_{h}, \mathbf{H}_{h}\right)$ be respectively the solutions of (2.1) and (2.10). Our goal is to estimate $\left\|\mathbf{E}-\mathbf{E}_{h}\right\|_{0, \Omega}$ and $\left\|\mathbf{H}-\mathbf{H}_{h}\right\|_{0, \Omega}$. For that, we introduce the energy norm:

$$
\|(\mathbf{E}, \mathbf{H})\|_{*}^{2}=\|\mathbf{E}\|_{0, \Omega, \underline{\underline{\underline{\varepsilon}}}}^{2}+\|\mathbf{H}\|_{0, \Omega, \underline{\underline{\mu}}}^{2} .
$$

The norm (4.1) is more adapted to our estimations because it appears naturally in the Maxwell equations. So, we prefer to estimate:

$$
\left\|\left(\mathbf{E}-\mathbf{E}_{h}, \mathbf{H}-\mathbf{H}_{h}\right)\right\|_{*}=\sqrt{\left\|\mathbf{E}-\mathbf{E}_{h}\right\|_{0, \Omega, \underline{\underline{\varepsilon}}}^{2}+\left\|\mathbf{H}-\mathbf{H}_{h}\right\|_{0, \Omega, \underline{\underline{\mu}}}^{2}} .
$$

Introduce the projection of the exact solution $(\mathbf{E}, \mathbf{H})$ i.e. $\left(\pi_{h}^{1} \mathbf{E}, \pi_{h}^{1} \mathbf{H}\right)$ (we assume that $\mathbf{E}$ and $\mathbf{H}$ have the regularity necessary for the definition of projections in (4.2)):

$$
\begin{gathered}
\left\|\left(\mathbf{E}-\mathbf{E}_{h}, \mathbf{H}-\mathbf{H}_{h}\right)\right\|_{*}^{2} \\
=\left\|\mathbf{E}-\pi_{h}^{1} \mathbf{E}+\pi_{h}^{1} \mathbf{E}-\mathbf{E}_{h}\right\|_{0, \Omega, \underline{\underline{\varepsilon}}}^{2}+\left\|\mathbf{H}-\pi_{h}^{1} \mathbf{H}+\pi_{h}^{1} \mathbf{H}-\mathbf{H}_{h}\right\|_{0, \Omega, \underline{\underline{\mu}}}^{2} \\
\leq\left\|\Delta_{\mathbf{E}}^{P}\right\|_{0, \Omega, \underline{\underline{\varepsilon}}}^{2}+\left\|\Delta_{\mathbf{E}}^{I}\right\|_{0, \Omega, \underline{\underline{\varepsilon}}}^{2}+2\left\|\Delta_{\mathbf{E}}^{P}\right\|_{0, \Omega, \underline{\underline{\varepsilon}}}\left\|\Delta_{\mathbf{E}}^{I}\right\|_{0, \Omega, \underline{\underline{\varepsilon}}} \\
+\left\|\Delta_{\mathbf{H}}^{P}\right\|_{0, \Omega, \underline{\underline{\mu}}}^{2}+\left\|\Delta_{\mathbf{H}}^{I}\right\|_{0, \Omega, \underline{\underline{\mu}}}^{2}+2\left\|\Delta_{\mathbf{H}}^{P}\right\|_{0, \Omega, \underline{\underline{\mu}}}\left\|\Delta_{\mathbf{H}}^{I}\right\|_{0, \Omega, \underline{\underline{\mu}}}
\end{gathered}
$$


where $\Delta_{\mathbf{E}}^{P}=\mathbf{E}-\pi_{h}^{1} \mathbf{E}$ (projection error) and $\Delta_{\mathbf{E}}^{I}=\mathbf{E}_{h}-\pi_{h}^{1} \mathbf{E}$ (interpolation error). We have the same thing for $\mathbf{H}$. Using the inequality $2 a b \leq a^{2}+b^{2}$, (4.3) becomes:

$$
\left\|\left(\mathbf{E}-\mathbf{E}_{h}, \mathbf{H}-\mathbf{H}_{h}\right)\right\|_{*}^{2} \leq 2\left(\left\|\left(\Delta_{\mathbf{E}}^{P}, \Delta_{\mathbf{H}}^{P}\right)\right\|_{*}^{2}+\left\|\left(\Delta_{\mathbf{E}}^{I}, \Delta_{\mathbf{H}}^{I}\right)\right\|_{*}^{2}\right) .
$$

To estimate the error introduced by the spatial approximation, we have to evaluate $\left\|\left(\Delta_{\mathbf{E}}^{P}, \Delta_{\mathbf{H}}^{P}\right)\right\|_{*}$ and $\left\|\left(\Delta_{\mathbf{E}}^{I}, \Delta_{\mathbf{H}}^{I}\right)\right\|_{*}$. The estimation of the first term does not pose any problem, it is sufficient to use the projection errors of the previous section; on the other hand, the second term requires more work. This will be done in three steps: first we will set up the equations which will make it possible to evaluate this error, then we will present two trace lemmas which will be used to estimate the surface integrals and finally we will consecutively evaluate the interpolation error for the study in $h$ and $r$.

4.1. Orthogonal property. Introducing $\left(\pi_{h}^{1} \mathbf{E}, \pi_{h}^{1} \mathbf{H}\right)$ in the semi-discrete DG system (without numerical integration) and taking $\phi_{1 h}=\Delta_{\mathbf{E}}^{I}$, we obtain:

$$
\begin{gathered}
\frac{1}{2} \frac{d}{d t}\left(\Delta_{\mathbf{E}}^{I}, \Delta_{\mathbf{E}}^{I}\right)_{0, K, \underline{\underline{\underline{\varepsilon}}}}=-\left(\frac{\partial}{\partial t} \pi_{h}^{1} \mathbf{E}, \Delta_{\mathbf{E}}^{I}\right)_{0, K, \underline{\underline{\underline{\varepsilon}}}}+\left(\nabla \times \Delta_{\mathbf{H}}^{I}, \Delta_{\mathbf{E}}^{I}\right)_{0, K} \\
+\left(\nabla \times \pi_{h}^{1} \mathbf{H}, \Delta_{\mathbf{E}}^{I}\right)_{0, K}-\left(\Delta_{\mathbf{E}}^{I}, \Delta_{\mathbf{E}}^{I}\right)_{0, K, \underline{\underline{\sigma}}}-\left(\pi_{h}^{1} \mathbf{E}, \Delta_{\mathbf{E}}^{I}\right)_{0, K, \underline{\underline{\sigma}}} \\
-\left(\mathbf{J}_{s}, \Delta_{\mathbf{E}}^{I}\right)_{0, K}+\left(\beta \llbracket \Delta_{\mathbf{H}}^{I} \times \mathbf{n}_{K} \rrbracket_{\partial K}^{K}, \Delta_{\mathbf{E} \mid K}^{I}\right)_{0, \partial K}+\left(\beta \llbracket \pi_{h}^{1} \mathbf{H} \times \mathbf{n}_{K} \rrbracket_{\partial K}^{K}, \Delta_{\mathbf{E} \mid K}^{I}\right)_{0, \partial K} .
\end{gathered}
$$

It is easy to see that the exact solution verifies:

$$
\begin{gathered}
\left(\frac{\partial}{\partial t} \Delta_{\mathbf{E}}^{P}, \Delta_{\mathbf{E}}^{I}\right)_{0, K, \underline{\underline{\varepsilon}}}+\left(\frac{\partial}{\partial t} \pi_{h}^{1} \mathbf{E}, \Delta_{\mathbf{E}}^{I}\right)_{0, K, \underline{\underline{\varepsilon}}}-\left(\nabla \times \Delta_{\mathbf{H}}^{P}, \Delta_{\mathbf{E}}^{I}\right)_{0, K} \\
-\left(\nabla \times \pi_{h}^{1} \mathbf{H}, \Delta_{\mathbf{E}}^{I}\right)_{0, K}+\left(\Delta_{\mathbf{E}}^{P}, \Delta_{\mathbf{E}}^{I}\right)_{0, K, \underline{\underline{\sigma}}} \\
+\left(\pi_{h}^{1} \mathbf{E}, \Delta_{\mathbf{E}}^{I}\right)_{0, K, \underline{\underline{\sigma}}}+\left(\mathbf{J}_{s}, \Delta_{\mathbf{E}}^{I}\right)_{0, K}=0 .
\end{gathered}
$$

Combine (4.5) and (4.6):

$$
\begin{aligned}
& \frac{1}{2} \frac{d}{d t}\left(\Delta_{\mathbf{E}}^{I}, \Delta_{\mathbf{E}}^{I}\right)_{0, K, \underline{\underline{\varepsilon}}}=-\left(\Delta_{\mathbf{E}}^{I}, \Delta_{\mathbf{E}}^{I}\right)_{0, K, \underline{\underline{\sigma}}}+\left(\frac{\partial}{\partial t} \Delta_{\mathbf{E}}^{P}, \Delta_{\mathbf{E}}^{I}\right)_{0, K, \underline{\underline{\varepsilon}}} \\
& +\left(\nabla \times \Delta_{\mathbf{H}}^{I}, \Delta_{\mathbf{E}}^{I}\right)_{0, K}-\left(\nabla \times \Delta_{\mathbf{H}}^{P}, \Delta_{\mathbf{E}}^{I}\right)_{0, K}+\left(\Delta_{\mathbf{E}}^{P}, \Delta_{\mathbf{E}}^{I}\right)_{0, K, \underline{\underline{\sigma}}} \\
& +\left(\beta \llbracket \Delta_{\mathbf{H}}^{I} \times \mathbf{n}_{K} \rrbracket_{\partial K}^{K}, \Delta_{\mathbf{E} \mid K}^{I}\right)_{0, \partial K}+\left(\beta \llbracket \pi_{h}^{1} \mathbf{H} \times \mathbf{n}_{K} \rrbracket_{\partial K}^{K}, \Delta_{\mathbf{E} \mid K}^{I}\right)_{0, \partial K} .
\end{aligned}
$$

Applying the same reasoning for the $\mathbf{H}$ equation, we have:

$$
\begin{gathered}
\frac{1}{2} \frac{d}{d t}\left(\Delta_{\mathbf{H}}^{I}, \Delta_{\mathbf{H}}^{I}\right)_{0, K, \underline{\underline{\mu}}}=\left(\frac{\partial}{\partial t} \Delta_{\mathbf{H}}^{P}, \Delta_{\mathbf{H}}^{I}\right)_{0, K, \underline{\underline{\mu}}}-\left(\nabla \times \Delta_{\mathbf{E}}^{I}, \Delta_{\mathbf{H}}^{I}\right)_{0, K} \\
+\left(\nabla \times \Delta_{\mathbf{E}}^{P}, \Delta_{\mathbf{H}}^{I}\right)_{0, K}+\left(\gamma \llbracket \Delta_{\mathbf{E}}^{I} \times \mathbf{n}_{K} \rrbracket_{\partial K}^{K}, \Delta_{\mathbf{H} \mid K}^{I}\right)_{0, \partial K} \\
+\left(\gamma \llbracket \pi_{h}^{1} \mathbf{E} \times \mathbf{n}_{K} \rrbracket_{\partial K}^{K}, \Delta_{\mathbf{H} \mid K}^{I}\right)_{0, \partial K} .
\end{gathered}
$$

The Green formula gives:

$$
\left(\nabla \times \Delta_{\mathbf{H}}^{I}, \Delta_{\mathbf{E}}^{I}\right)_{0, K}=\left(\Delta_{\mathbf{H}}^{I}, \nabla \times \Delta_{\mathbf{E}}^{I}\right)_{0, K}+\left(\Delta_{\mathbf{H} \mid K}^{I}, \Delta_{\mathbf{E} \mid K}^{I} \times \mathbf{n}_{K}\right)_{0, \partial K} .
$$


Adding (4.7) and (4.8), we obtain:

$$
\begin{gathered}
\frac{1}{2} \frac{d}{d t}\left[\left(\Delta_{\mathbf{E}}^{I}, \Delta_{\mathbf{E}}^{I}\right)_{0, K, \underline{\underline{\varepsilon}}}+\left(\Delta_{\mathbf{H}}^{I}, \Delta_{\mathbf{H}}^{I}\right)_{0, K, \underline{\underline{\mu}}}\right] \\
=\left[\left(\frac{\partial}{\partial t} \Delta_{\mathbf{E}}^{P}, \Delta_{\mathbf{E}}^{I}\right)_{0, K, \underline{\underline{\varepsilon}}}+\left(\frac{\partial}{\partial t} \Delta_{\mathbf{H}}^{P}, \Delta_{\mathbf{H}}^{I}\right)_{0, K, \underline{\underline{\mu}}}\right]+\left(\nabla \times \Delta_{\mathbf{E}}^{P}, \Delta_{\mathbf{H}}^{I}\right)_{0, K} \\
-\left(\nabla \times \Delta_{\mathbf{H}}^{P}, \Delta_{\mathbf{E}}^{I}\right)_{0, K}-\left(\Delta_{\mathbf{E}}^{I}, \Delta_{\mathbf{E}}^{I}\right)_{0, K, \underline{\underline{\sigma}}}+\left(\Delta_{\mathbf{E}}^{P}, \Delta_{\mathbf{E}}^{I}\right)_{0, K, \underline{\underline{\sigma}}} \\
+\left(\beta \llbracket \Delta_{\mathbf{H}}^{I} \times \mathbf{n}_{K} \rrbracket_{\partial K}^{K}, \Delta_{\mathbf{E} \mid K}^{I}\right)_{0, \partial K}+\left(\beta \llbracket \pi_{h}^{1} \mathbf{H} \times \mathbf{n}_{K} \rrbracket_{\partial K}^{K}, \Delta_{\mathbf{E} \mid K}^{I}\right)_{0, \partial K} \\
+\left(\Delta_{\mathbf{H} \mid K}^{I}, \Delta_{\mathbf{E} \mid K}^{I} \times \mathbf{n}_{K}\right)_{0, \partial K}-\left(\gamma \llbracket \Delta_{\mathbf{E}}^{I} \times \mathbf{n}_{K} \rrbracket_{\partial K}^{K}, \Delta_{\mathbf{H} \mid K}^{I}\right)_{0, \partial K} \\
+\left(\gamma \llbracket \pi_{h}^{1} \mathbf{E} \times \mathbf{n}_{K} \rrbracket_{\partial K}^{K}, \Delta_{\mathbf{H} \mid K}^{I}\right)_{0, \partial K} .
\end{gathered}
$$

We know that $\forall t \in(0, T),(\mathbf{E}, \mathbf{H})(t) \in H_{0}($ rot,$\Omega) \times H($ rot,$\Omega)$, so we have $\forall \Gamma=$ $\left(K \cap K^{\prime}\right) \in \mathscr{F}_{h}^{i}, \llbracket \mathbf{E} \times \mathbf{n}_{K} \rrbracket_{\Gamma}^{K}$ ou $K^{\prime}=0$ and $\llbracket \mathbf{H} \times \mathbf{n}_{K} \rrbracket_{\Gamma}^{K}$ ou $K^{\prime}=0$. Moreover, keep in mind that $\forall \Gamma \in \mathscr{F}_{h}^{b}, \beta=0$.

By summing (4.10) over all the cells of the mesh and using the previous properties, we can write:

$$
\begin{gathered}
\frac{1}{2} \frac{d}{d t}\left\|\left(\Delta_{E}^{I}, \Delta_{H}^{I}\right)\right\|_{*}^{2}=\frac{1}{2} \frac{d}{d t} \sum_{K \in \mathscr{T}_{h}}\left[\left(\Delta_{\mathbf{E}}^{I}, \Delta_{\mathbf{E}}^{I}\right)_{0, K, \underline{\underline{\underline{\varepsilon}}}}+\left(\Delta_{\mathbf{H}}^{I}, \Delta_{\mathbf{H}}^{I}\right)_{0, K, \underline{\underline{\mu}}}\right] \\
\leq \sum_{K \in \mathscr{T}_{h}}\left[\left|\left(\frac{\partial}{\partial t} \Delta_{\mathbf{E}}^{P}, \Delta_{\mathbf{E}}^{I}\right)_{0, K, \underline{\underline{\varepsilon}}}\right|+\left|\left(\frac{\partial}{\partial t} \Delta_{\mathbf{H}}^{P}, \Delta_{\mathbf{H}}^{I}\right)_{0, K, \underline{\underline{\mu}}}\right|+\left|\left(\nabla \times \Delta_{\mathbf{E}}^{P}, \Delta_{\mathbf{H}}^{I}\right)_{0, K}\right|\right. \\
+\left|\left(\nabla \times \Delta_{\mathbf{H}}^{P}, \Delta_{\mathbf{E}}^{I}\right)_{0, K}\right|+\left|\left(\Delta_{\mathbf{E}}^{P}, \Delta_{\mathbf{E}}^{I}\right)_{0, K, \underline{\underline{\sigma}}}\right|+\left|\left(\beta \llbracket \Delta_{\mathbf{H}}^{P} \times \mathbf{n}_{K} \rrbracket, \Delta_{\mathbf{E}, K}^{I}\right)_{0, \partial K}\right| \\
\left.+\left|\left(\gamma \llbracket \Delta_{\mathbf{E}}^{P} \times \mathbf{n}_{K} \rrbracket, \Delta_{\mathbf{H}, K}^{I}\right)_{0, \partial K}\right|\right]
\end{gathered}
$$

To obtain (4.11), we have used the fact that $\left(\Delta_{\mathbf{E}}^{I}, \Delta_{\mathbf{E}}^{I}\right)_{0, K, \underline{\underline{\sigma}}} \geq 0$ and to eliminate surface terms in $\Delta_{\mathbf{E}}^{I}$ and $\Delta_{\mathbf{H}}^{I}$, we have used the identity:

$$
\begin{gathered}
\sum_{K \in \mathscr{T}_{h}}\left(\left(\beta \llbracket \Delta_{\mathbf{H}}^{I} \times \mathbf{n}_{K} \rrbracket_{\partial K}^{K}, \Delta_{\mathbf{E} \mid K}^{I}\right)_{0, \partial K}-\left(\gamma \llbracket \Delta_{\mathbf{E}}^{I} \times \mathbf{n}_{K} \rrbracket_{\partial K}^{K}, \Delta_{\mathbf{H} \mid K}^{I}\right)_{0, \partial K}\right. \\
\left.+\left(\Delta_{\mathbf{H} \mid K}^{I}, \Delta_{\mathbf{E} \mid K}^{I} \times \mathbf{n}_{K}\right)_{0, \partial K}\right)=0
\end{gathered}
$$

4.2. Trace lemmas. To estimate the surface integrals, we will need several intermediate results:

Lemma 4.1. Let $\mathbf{u}_{h} \in U_{h}$ and $K \in \mathscr{T}_{h}$; then there exists a constant $C>0$ independent of $K$ and $r$ such that:

$$
\left(\mathbf{u}_{h \mid K}, \mathbf{u}_{h \mid K}\right)_{0, \partial K} \leq C \sigma_{K}^{11} \frac{r^{2}}{\rho_{K}}\left(\mathbf{u}_{h}, \mathbf{u}_{h}\right)_{0, K} .
$$


Moreover, if $\mathscr{T}_{h}$ belongs to a regular family of meshes, we have:

$$
\left(\mathbf{u}_{h \mid K}, \mathbf{u}_{h \mid K}\right)_{0, \partial K} \leq C \frac{r^{2}}{h_{K}}\left(\mathbf{u}_{h}, \mathbf{u}_{h}\right)_{0, K} .
$$

Proof. We have:

$$
\frac{\int_{\partial K}\left|\mathbf{u}_{h K}\right|^{2} d \sigma}{\int_{K}\left|\mathbf{u}_{h K}\right|^{2} d x}=\frac{\int_{\partial \hat{K}}\left|J_{K}\right||| D F_{K}^{*-1} \hat{n} \|\left(D F_{K}^{-1} D F_{K}^{*-1} \hat{\mathbf{u}}_{K}\right) \cdot \hat{\mathbf{u}}_{K} d \hat{\sigma}}{\int_{\hat{K}}\left|J_{K}\right|\left(D F_{K}^{-1} D F_{K}^{*-1} \hat{\mathbf{u}}_{K}\right) \cdot \hat{\mathbf{u}}_{K} d \hat{x}} .
$$

The estimations (3.3) lead to:

$$
\frac{\int_{\partial K}\left|\mathbf{u}_{h K}\right|^{2} d \sigma}{\int_{K}\left|\mathbf{u}_{h K}\right|^{2} d x} \leq C \frac{\sigma_{K}^{11}}{\rho_{K}} \frac{\int_{\partial \hat{K}} \hat{\mathbf{u}}_{K} \cdot \hat{\mathbf{u}}_{K} d \hat{\sigma}}{\int_{\hat{K}} \hat{\mathbf{u}}_{K} \cdot \hat{\mathbf{u}}_{K} d \hat{x}}
$$

In [29], we can find the estimation:

$$
\frac{\int_{\partial \hat{K}} \hat{\mathbf{u}}_{K} \cdot \hat{\mathbf{u}}_{K} d \hat{\sigma}}{\int_{\hat{K}} \hat{\mathbf{u}}_{K} \cdot \hat{\mathbf{u}}_{K} d \hat{x}} \leq C r^{2} .
$$

So, we obtain the wanted result.

We will need the trace inequality also:

Lemma 4.2. Let $K \in \mathscr{T}_{h}$. There exists $C>0$ independent of $K$ and $r$ such that $\forall v \in H^{1}(K)$,

$$
\|v\|_{0, \partial K}^{2} \leq C\left(\|v\|_{0, K}\|\nabla v\|_{0, K}+\rho_{K}^{-1} \sigma_{K}^{-1}\|v\|_{0, K}^{2}\right)
$$

Moreover, if $\mathscr{T}_{h}$ belongs to a regular family of meshes, we have:

$$
\|v\|_{0, \partial K}^{2} \leq C\left(\|v\|_{0, K}\|\nabla v\|_{0, K}+h_{K}^{-1}\|v\|_{0, K}^{2}\right) .
$$

Proof. Let $K \in \mathscr{T}_{h}$ and $\mathbf{v} \in H^{1}(K)$. Pose $\hat{v}=v o F_{K}$. So, we have the trace inequality:

$$
\|\hat{v}\|_{0, \partial \hat{K}}^{2} \leq C\left(\|\hat{v}\|_{0, \hat{K}}\|\hat{\nabla} \hat{v}\|_{0, \hat{K}}+\|\hat{v}\|_{0, \hat{K}}^{2}\right) .
$$

See for example the annexes of [28] to obtain a proof of this result. 
Now, we are going to return to the cell $K$. We have the estimations:

$$
\begin{aligned}
& \bullet\|\hat{v}\|_{0, \partial \hat{K}}^{2}=\int_{\partial \hat{K}} \hat{v}^{2} d \hat{\sigma}=\int_{\partial K} \frac{1}{\left|J_{K}\right|\left\|D F_{K}^{*-1} \hat{\mathbf{n}}\right\|} v^{2} d \sigma \\
& \geq \frac{1}{\left\|J_{K}\right\|_{\infty, \hat{K}}\left|F_{K}^{-1}\right|_{1, \infty, K}}\|v\|_{0, \partial K}^{2} \\
& \geq C \frac{\sigma_{K}^{3}}{h_{K}^{2}}\|v\|_{0, \partial K}^{2} \text { by using (3.3), } \\
& \bullet\|\hat{v}\|_{0, \hat{K}}^{2}=\int_{\hat{K}} \hat{v}^{2} d \hat{\mathbf{x}}=\int_{K} \frac{1}{\left|J_{K}\right|} v^{2} d \mathbf{x} \leq\left\|J_{K}^{-1}\right\|_{\infty, K}\|v\|_{0, K}^{2}=\rho_{K}^{-3}\|v\|_{0, K}^{2} \text {, } \\
& \bullet\|\hat{\nabla} \hat{v}\|_{0, \hat{K}}^{2}=\int_{\hat{K}} \hat{\nabla} \hat{v} \cdot \hat{\nabla} \hat{v} d \hat{\mathbf{x}}=\int_{K} \frac{1}{\left|J_{K}\right|} D F_{K}^{*} \nabla v \cdot D F_{K}^{*} \nabla v d \mathbf{x} \\
& \leq C \frac{\sigma_{K}^{2}}{\rho_{K}}\|\nabla v\|_{0, K}^{2} \text { by using (3.3). }
\end{aligned}
$$

(4.20) becomes:

$$
C_{1} \frac{\sigma_{K}^{3}}{h_{K}^{2}}\|v\|_{0, \partial K}^{2} \leq C\left(C_{2} \frac{\sigma_{K}}{\rho_{K}^{2}}\|v\|_{0, K}\|\nabla v\|_{0, K}+C_{3} \frac{1}{\rho_{K}^{3}}\|v\|_{0, K}^{2}\right) .
$$

We obtain the wanted result.

4.3. Error estimates. The use of (4.12) allows us to establish the following estimations of the surface terms:

$$
\begin{gathered}
\sum_{K \in \mathscr{T}_{h}}\left[\left|\left(\beta \llbracket \Delta_{\mathbf{H}}^{P} \times \mathbf{n}_{K} \rrbracket, \Delta_{\mathbf{E} \mid K}^{I}\right)_{0, \partial K}\right|+\left|\left(\gamma \llbracket \Delta_{\mathbf{E}}^{P} \times \mathbf{n}_{K} \rrbracket, \Delta_{\mathbf{H} \mid K}^{I}\right)_{0, \partial K}\right|\right] \\
\leq \sum_{K \in \mathscr{T}_{h}}\left[|\beta|\left\|\llbracket \Delta_{\mathbf{H}}^{P} \times \mathbf{n}_{K} \rrbracket\right\|_{0, \partial K}\left\|\Delta_{\mathbf{E} \mid K}^{I}\right\|_{0, \partial K}\right. \\
\left.\quad+|\gamma|\left\|\llbracket \Delta_{\mathbf{E}}^{P} \times \mathbf{n}_{K} \rrbracket\right\|_{0, \partial K}\left\|\Delta_{\mathbf{H} \mid K}^{I}\right\|_{0, \partial K}\right] \\
\leq C \sum_{K \in \mathscr{T}_{h}} \sigma_{K}^{\frac{11}{2}} \frac{r}{\rho_{K}^{\frac{1}{2}}}\left[|\beta|\left\|\llbracket \Delta_{\mathbf{H}}^{P} \times \mathbf{n}_{K} \rrbracket\right\|_{0, \partial K}\left\|\Delta_{\mathbf{E}}^{I}\right\|_{0, K, \underline{\underline{\varepsilon}}}\right. \\
\left.\quad+|\gamma| \mid \llbracket \llbracket \Delta_{\mathbf{E}}^{P} \times \mathbf{n}_{K} \rrbracket\left\|_{0, \partial K}\right\| \Delta_{\mathbf{H}}^{I} \|_{0, K, \mu}\right]
\end{gathered}
$$

where $C$ is a constant independent of $K$ and $r$, but dependent on materials in the event here of $B_{1}$ defined in the first section. 
(4.17) gives:

$$
\begin{aligned}
& \left\|\Delta_{\mathbf{E} \mid K}^{P} \times \mathbf{n}_{K}\right\|_{0, \partial K}^{2} \leq C\left(\left\|\Delta_{\mathbf{E}}^{P}\right\|_{0, K}\left\|\Delta_{\mathbf{E}}^{P}\right\|_{1, K}+\sigma_{K}^{-1} \rho_{K}^{-1}\left\|\Delta_{\mathbf{E}}^{P}\right\|_{0, K}^{2}\right), \\
& \left\|\Delta_{\mathbf{H} \mid K}^{P} \times \mathbf{n}_{K}\right\|_{0, \partial K}^{2} \leq C\left(\left\|\Delta_{\mathbf{H}}^{P}\right\|_{0, K}\left\|\Delta_{\mathbf{H}}^{P}\right\|_{1, K}+\sigma_{K}^{-1} \rho_{K}^{-1}\left\|\Delta_{\mathbf{H}}^{P}\right\|_{0, K}^{2}\right)
\end{aligned}
$$

where $C$ is a constant independent of $K$ and $r$.

Indeed, note that $\mathbf{v}=\Delta_{\mathbf{E} \mid K}^{P}=\left(v_{1}, v_{2}, v_{3}\right)^{*}$ and $\mathbf{n}_{K}=\left(n_{1}, n_{2}, n_{3}\right)^{*}$. We can then write:

$$
\Delta_{\mathbf{E} \mid K}^{P} \times \mathbf{n}_{K}=\mathbf{v} \times \mathbf{n}_{K}=\left(v_{2} n_{3}-v_{3} n_{2}, v_{3} n_{1}-v_{1} n_{3}, v_{1} n_{2}-v_{2} n_{1}\right)^{*} .
$$

Now, developing $\left\|\Delta_{\mathbf{E} \mid K}^{P} \times \mathbf{n}_{K}\right\|_{0, \partial K}^{2}$, we get:

$$
\begin{gathered}
\left\|\Delta_{\mathbf{E} \mid K}^{P} \times \mathbf{n}_{K}\right\|_{0, \partial K}^{2} \\
=\int_{\partial K}\left(\left(v_{2} n_{3}-v_{3} n_{2}\right)^{2}+\left(v_{3} n_{1}-v_{1} n_{3}\right)^{2}+\left(v_{1} n_{2}-v_{2} n_{1}\right)^{2}\right) d \sigma \\
\leq 2 \int_{\partial K}\left(v_{2}^{2} n_{3}^{2}+v_{3}^{2} n_{2}^{2}+v_{3}^{2} n_{1}^{2}+v_{1}^{2} n_{3}^{2}+v_{1}^{2} n_{2}^{2}+v_{2}^{2} n_{1}^{2}\right) d \sigma \\
\leq 4 \int_{\partial K}\left(v_{1}^{2}+v_{2}^{2}+v_{3}^{2}\right) d \sigma=4\left(\left\|v_{1}\right\|_{0, \partial K}^{2}+\left\|v_{2}\right\|_{0, \partial K}^{2}+\left\|v_{3}\right\|_{0, \partial K}^{2}\right) .
\end{gathered}
$$

To obtain the last inequality, we have used the fact that $n_{1}^{2}+n_{2}^{2}+n_{3}^{2}=1$. Applying (4.18) to $v_{i} \in H^{1}(K)$ (for $1 \leq i \leq 3$ ), one deduces the inequalities:

$$
\left\|v_{i}\right\|_{0, \partial K}^{2} \leq C\left(\left\|v_{i}\right\|_{0, K}\left\|\nabla v_{i}\right\|_{0, K}+\sigma_{K}^{-1} \rho_{K}^{-1}\left\|v_{i}\right\|_{0, K}^{2}\right)
$$

Finally, introducing (4.28) into (4.27), we have:

$$
\begin{aligned}
\left\|\Delta_{\mathbf{E} \mid K}^{P} \times \mathbf{n}_{K}\right\|_{0, \partial K}^{2} \leq & 4 C \sum_{i=1}^{3}\left(\left\|v_{i}\right\|_{0, K}\left\|\nabla v_{i}\right\|_{0, K}+\sigma_{K}^{-1} \rho_{K}^{-1}\left\|v_{i}\right\|_{0, K}^{2}\right) \\
& \leq 4 C \sum_{i=1}^{3}\left(\|\mathbf{v}\|_{0, K}\|\mathbf{v}\|_{1, K}+\sigma_{K}^{-1} \rho_{K}^{-1}\|\mathbf{v}\|_{0, K}^{2}\right) \\
& \leq 12 C\left(\|\mathbf{v}\|_{0, K}\|\mathbf{v}\|_{1, K}+\sigma_{K}^{-1} \rho_{K}^{-1}\|\mathbf{v}\|_{0, K}^{2}\right) .
\end{aligned}
$$

We obtain the wanted result. For $\left\|\Delta_{\mathbf{H} \mid K}^{P} \times \mathbf{n}_{K}\right\|_{0, \partial K}^{2}$ it is obviously the same thing. 
For the other terms of (4.11), we have the following estimates:

$$
\begin{aligned}
& \cdot \sum_{K \in \mathscr{T}_{h}}\left[\left|\left(\nabla \times \Delta_{\mathbf{E}}^{P}, \Delta_{\mathbf{H}}^{I}\right)_{0, K}\right|+\left|\left(\nabla \times \Delta_{\mathbf{H}}^{P}, \Delta_{\mathbf{E}}^{I}\right)_{0, K}\right|\right] \\
& \leq C \sum_{K \in \mathscr{T}_{h}}\left[\left\|\Delta_{\mathbf{E}}^{P}\right\|_{1, K}\left\|\Delta_{\mathbf{H}}^{I}\right\|_{0, K, \underline{\underline{\mu}}}+\left\|\Delta_{\mathbf{H}}^{P}\right\|_{1, K}\left\|\Delta_{\mathbf{E}}^{I}\right\|_{0, K, \underline{\underline{\varepsilon}}}\right], \\
& \text { - } \sum_{K \in \mathscr{T}_{h}}\left|\left(\frac{\partial}{\partial t} \Delta_{\mathbf{E}}^{P}, \Delta_{\mathbf{E}}^{I}\right)_{0, K, \underline{\underline{\varepsilon}}}\right|+\left|\left(\frac{\partial}{\partial t} \Delta_{\mathbf{H}}^{P}, \Delta_{\mathbf{H}}^{I}\right)_{0, K, \underline{\underline{\mu}}}\right| \\
& \leq C \sum_{K \in \mathscr{T}_{h}}\left(\left\|\Delta_{\mathbf{E}_{t}}^{P}\right\|_{0, K}\left\|\Delta_{\mathbf{E}}^{I}\right\|_{0, K, \underline{\underline{\varepsilon}}}+\left\|\Delta_{\mathbf{H}_{t}}^{P}\right\|_{0, K}\left\|\Delta_{\mathbf{H}}^{I}\right\|_{0, K, \underline{\underline{\varepsilon}}},\right. \\
& \text { - } \sum_{K \in \mathscr{T}_{h}}\left|\left(\Delta_{\mathbf{E}}^{P}, \Delta_{\mathbf{E}}^{I}\right)_{0, K, \underline{\underline{\sigma}}}\right| \leq C \sum_{K \in \mathscr{T}_{h}}\left\|\Delta_{\mathbf{E}}^{P}\right\|_{0, K}\left\|\Delta_{\mathbf{E}}^{I}\right\|_{0, K, \underline{\underline{\varepsilon}}}
\end{aligned}
$$

where $C$ is a constant independent of $K$ and $r$ but dependent on the dielectric values of the medium and $\mathbf{u}_{t}=\frac{\partial}{\partial t} \mu$.

Remark 4.3. To obtain the second inequality of (4.30), we have used the property:

$$
\frac{\partial}{\partial t} \Delta_{\mathbf{E}}^{P}=\Delta_{\frac{\partial}{\partial t} \mathbf{E}}^{P}=\Delta_{\mathbf{E}_{t}}^{P} .
$$

We have the same thing for $\Delta_{\mathbf{H}}^{P}$.

Now, we are going to recombine the established estimates and use the projection errors of the previous section. Keep in mind that we use a regular family, $\left(\mathscr{T}_{h}\right)_{h>0}$, of meshes. We assume that $\mathbf{E}, \mathbf{H} \in \mathbf{H}^{s+1}\left(\mathscr{T}_{h}\right) \cap H(\operatorname{rot}, \Omega), \mathbf{E}_{t}, \mathbf{H}_{t} \in \mathbf{H}^{s^{\prime}+1}\left(\mathscr{T}_{h}\right)$ and $\mathbf{J}_{s} \in \mathbf{H}^{s^{\prime \prime}+1}\left(\mathscr{T}_{h}\right)$ with $0 \leq s, s^{\prime}, s^{\prime \prime} \leq r$ and $0<h_{K} \leq 1, \forall K \in \mathscr{T}_{h}$.

Using (3.46), (4.25) becomes:

$$
\begin{aligned}
& \left\|\Delta_{\mathbf{E} \mid K}^{P} \times \mathbf{n}_{K}\right\|_{0, \partial K}^{2} \leq C \frac{h_{K}^{\min (2 s-1,2 r-1)}}{r^{2 s+1}}\|\mathbf{E}\|_{s+1, K}, \\
& \left\|\Delta_{\mathbf{H} \mid K}^{P} \times \mathbf{n}_{K}\right\|_{0, \partial K}^{2} \leq C \frac{h_{K}^{\min (2 s-1,2 r-1)}}{r^{2 s+1}}\|\mathbf{H}\|_{s+1, K} .
\end{aligned}
$$

Thus, the boundary terms are bounded by:

$$
\begin{gathered}
\sum_{K \in \mathscr{T}_{h}}\left[\left|\left(\beta \llbracket \Delta_{\mathbf{H}}^{P} \times \mathbf{n}_{K} \rrbracket, \Delta_{\mathbf{E}, K}^{I}\right)_{0, \partial K}\right|+\left|\left(\gamma \llbracket \Delta_{\mathbf{E}}^{P} \times \mathbf{n}_{K} \rrbracket, \Delta_{\mathbf{H}, K}^{I}\right)_{0, \partial K}\right|\right] \\
\leq C \sum_{K \in \mathscr{T}_{h}} \frac{h_{K}^{\min (s-1, r-1)}}{r^{s-\frac{1}{2}}}\left[\|\mathbf{E}\|_{s+1, K}\left\|\Delta_{\mathbf{E}, K}^{I}\right\|_{0, K, \underline{\underline{\varepsilon}}}+\|\mathbf{H}\|_{s+1, K}\left\|\Delta_{\mathbf{H}, K}^{I}\right\|_{0, K, \underline{\underline{\mu}}}\right] .
\end{gathered}
$$

Here $C$ depends on $r$. 
We also have the estimates of (4.30):

$$
\begin{aligned}
& \cdot \sum_{K \in \mathscr{T}_{h}}\left[\left|\left(\nabla \times \Delta_{\mathbf{E}}^{P}, \Delta_{\mathbf{H}}^{I}\right)_{0, K}\right|+\left|\left(\nabla \times \Delta_{\mathbf{H}}^{P}, \Delta_{\mathbf{E}}^{I}\right)_{0, K}\right|\right] \\
& \leq C \sum_{K \in \mathscr{T}_{h}} \frac{h_{K}^{\min (s-1, r-1)}}{r^{s}}\left[\|\mathbf{E}\|_{s+1, K}\left\|\Delta_{\mathbf{H}}^{I}\right\|_{0, K, \underline{\underline{\mu}}}+\|\mathbf{H}\|_{s+1, K}\left\|\Delta_{\mathbf{E}}^{I}\right\|_{0, K, \underline{\underline{\varepsilon}}}\right] \\
& \text { - } \sum_{K \in \mathscr{T}_{h}}\left|\left(\frac{\partial}{\partial t} \Delta_{\mathbf{E}}^{P}, \Delta_{\mathbf{E}}^{I}\right)_{0, K, \underline{\underline{\varepsilon}}}\right|+\left|\left(\frac{\partial}{\partial t} \Delta_{\mathbf{H}}^{P}, \Delta_{\mathbf{H}}^{I}\right)_{0, K, \underline{\underline{\mu}}}\right| \\
& \leq C \sum_{K \in \mathscr{T}_{h}} \frac{h_{K}^{\min \left(s^{\prime}, r\right)}}{r^{s^{\prime}+1}}\left[\left\|\mathbf{E}_{t}\right\|_{s^{\prime}+1, K}\left\|\Delta_{\mathbf{E}}^{I}\right\|_{0, K, \underline{\underline{\varepsilon}}}+\left\|\mathbf{H}_{t}\right\|_{s^{\prime}+1, K}\left\|\Delta_{\mathbf{H}}^{I}\right\|_{0, K, \underline{\underline{\mu}}}\right] \\
& \text { - } \sum_{K \in \mathscr{T}_{h}}\left|\left(\Delta_{\mathbf{E}}^{P}, \Delta_{\mathbf{E}}^{I}\right)_{0, K, \underline{\underline{\sigma}}}\right| \leq C \sum_{K \in \mathscr{T}_{h}} \frac{h_{K}^{\min (s, r)}}{r^{s+1}}\|\mathbf{E}\|_{s+1, K}\left\|\Delta_{\mathbf{E}}^{I}\right\|_{0, K, \underline{\underline{\varepsilon}}} .
\end{aligned}
$$

Now, by using the fact that $\frac{\left\|\Delta_{\mathbf{E}}^{I}\right\|_{0, K, \underline{\underline{\varepsilon}}}}{\left\|\left(\Delta_{E}^{I}, \Delta_{H}^{I}\right)\right\|_{*}} \leq 1$ and $\frac{\left\|\Delta_{\mathbf{H}}^{I}\right\|_{0, K, \underline{\underline{\mu}}}}{\left\|\left(\Delta_{E}^{I}, \Delta_{H}^{I}\right)\right\|_{*}} \leq 1$, (4.11) leads to:

$$
\begin{gathered}
\frac{d}{d t}\left\|\left(\Delta_{E}^{I}, \Delta_{H}^{I}\right)\right\|_{*} \leq C \sum_{K \in \mathscr{T}_{h}}\left[\frac{h_{K}^{\min (s-1, r-1)}}{r^{s-\frac{1}{2}}}\left(\|\mathbf{E}\|_{s+1, K}+\|\mathbf{H}\|_{s+1, K}\right)\right. \\
\left.+\frac{h_{K}^{\min \left(s^{\prime}, r\right)}}{r^{s^{\prime}+1}}\left(\left\|\mathbf{E}_{t}\right\|_{s^{\prime}+1, K}+\left\|\mathbf{H}_{t}\right\|_{s^{\prime}+1, K}\right)\right] .
\end{gathered}
$$

Finally, the Gronwall lemma on the interval $(0, T)$ gives the following theorem:

Theorem 4.4. Let $r$ be a positive integer. Assume that the exact solution verifies $(\mathbf{E}, \mathbf{H}) \in \mathbf{H}^{s+1}\left(\mathscr{T}_{h}\right)$ and $\left(\mathbf{E}_{t}, \mathbf{H}_{t}\right) \in \mathbf{H}^{s^{\prime}+1}\left(\mathscr{T}_{h}\right)$ for $s, s^{\prime} \geq 0$ real and $0<h_{K} \leq 1, \forall K \in \mathscr{T}_{h}$. Then,we have the global estimate of the interpolation error:

$$
\begin{gathered}
\left\|\left(\Delta_{E}^{I}, \Delta_{H}^{I}\right)\right\|_{*}(T) \leq\left\|\left(\Delta_{E}^{I}, \Delta_{H}^{I}\right)\right\|_{*}(0) \\
+C T \frac{h^{\min \left(s-1, s^{\prime}, r-1\right)}}{r^{\min \left(s-\frac{1}{2}, s^{\prime}+1\right)}} \max _{t \in(0, T)}\left(\|\mathbf{E}\|_{s+1, h}(t),\|\mathbf{H}\|_{s+1, h}(t),\right. \\
\left.\cdot\left\|\mathbf{E}_{t}\right\|_{s^{\prime}+1, h}(t),\left\|\mathbf{H}_{t}\right\|_{s^{\prime}+1, h}(t)\right)
\end{gathered}
$$

where $C>0$ is a constant independent of $K$ and $r$ and $h=\max h_{K}$. 
Return to the error of the scheme: According to (4.4), we have

$$
\begin{aligned}
& \left\|\left(\mathbf{E}-\mathbf{E}_{h}, \mathbf{H}-\mathbf{H}_{h}\right)\right\|_{*}(T) \leq \sqrt{2}\left(\left\|\left(\Delta_{\mathbf{E}}^{P}, \Delta_{\mathbf{H}}^{P}\right)\right\|_{*}+\left\|\left(\Delta_{\mathbf{E}}^{I}, \Delta_{\mathbf{H}}^{I}\right)\right\|_{*}\right)(T) \\
& \leq \sqrt{2}\left\|\left(\Delta_{E}^{I}, \Delta_{H}^{I}\right)\right\|_{*}(0)+C \sqrt{2}\left[h^{s} \max \left(\|\mathbf{E}\|_{s+1, h}(T),\|\mathbf{H}\|_{s+1, h}(T)\right)\right. \\
& \left.+T \frac{h^{\min \left(s-1, s^{\prime}, r-1\right)}}{r^{\min \left(s-\frac{1}{2}, s^{\prime}+1\right)}} \max _{t \in(0, T)}\left(\|\mathbf{E}\|_{s+1, h}(t),\|\mathbf{H}\|_{s+1, h}(t),\left\|\mathbf{E}_{t}\right\|_{s^{\prime}+1, h}(t),\left\|\mathbf{H}_{t}\right\|_{s^{\prime}+1, h}(t)\right)\right] .
\end{aligned}
$$

We see that the error seems to be sub-optimal and it increases at most linearly in time. Moreover, for $r=1$, the previous estimate does not prove the consistence of the scheme. In the last section of this paper, we will see with a simple numerical example that it is not clear that this scheme is consistent for a certain type of mesh.

Remark 4.5. If the mesh used is orthogonal or almost parallelepipedic, we find an exponent $h^{s}$. Indeed, we are respectively in an affine case and with second derivatives of $F_{K}$ bounded by $C h_{K}^{2}$.

4.4. Error due to the numerical integration. In this sub-section, we assume that the dielectric tensors are constant by cells and that we have conformal meshes. This last assumption allows us to have all discrete jump integrals (i.e. computed by using the Gauss rule) which are exact [34 i.e. $\forall \mathbf{u}_{h}, \mathbf{v}_{h} \in U_{h}$ we have:

$$
\int_{\partial K}^{G} \llbracket \mathbf{u}_{h} \times \mathbf{n}_{K} \rrbracket \cdot \mathbf{v}_{h} d \sigma=\int_{\partial K} \llbracket \mathbf{u}_{h} \times \mathbf{n}_{K} \rrbracket \cdot \mathbf{v}_{h} d \sigma .
$$

For technical reasons due to the use of a quadrature formula, we will need the interpolation operator $I_{h}$ on $U_{h}$ defined by: Let $\mathbf{v} \in\left[C^{0}\left(\mathscr{T}_{h}\right)\right]^{3}$ (i.e. $\mathbf{v} \in \mathbf{L}^{2}(\Omega)$ be such that $\forall K \in \mathscr{T}_{h}$ we have $\left.\mathbf{v}_{\mid K} \in\left[C^{0}(K)\right]^{3}\right)$; then $\forall K \in \mathscr{T}_{h}$,

$$
I_{h \mid K} \mathbf{v} o F_{K}\left(\boldsymbol{\xi}_{1}\right)=\mathbf{v} o F_{K}\left(\boldsymbol{\xi}_{1}\right)
$$

$\forall \mathbf{l} \in\{1, \cdots, r+1\}^{3}$. We can easily transpose the error estimates of the operator $\pi_{h}^{1}$ to $I_{h}$ and we obtain, in particular: Let $\mathbf{v} \in \mathbf{H}^{s+1}(K), s>\frac{1}{2}\left(s>\frac{3}{2}\right.$ to ensure the inclusion of $\mathbf{H}^{s}(K)$ in $\left.\left[C^{0}(K)\right]^{3}\right)$; then there exists $C$ independent of the element $K$ and $r$ such that:

$$
\left\|\mathbf{v}-I_{h \mid K} \mathbf{v}\right\|_{0, K} \leq C \frac{h_{K}^{\min (s, r)}}{r^{s+1}}\|\mathbf{v}\|_{s+1, K}
$$

To prove the $r$-dependence of (4.39), we have used the result in 31, $\mathbf{v} \in \mathbf{H}^{s+1}(K), s>\frac{1}{2}$, then there exists a constant $C$ independent of $r$ such that:

$$
\left\|\mathbf{v}-I_{h \mid K} \mathbf{v}\right\|_{0, K} \leq \frac{C}{r^{s+1}}\|\mathbf{v}\|_{s+1, K} .
$$


Now, let us rewrite equation (4.10) by taking into account the Gauss quadrature rule:

$$
\begin{aligned}
& \frac{1}{2} \frac{d}{d t} \int_{K}^{G}\left[\underline{\underline{\varepsilon}} \Delta_{I}^{\mathbf{E}} \cdot \Delta_{I}^{\mathbf{E}}+\underline{\mu} \Delta_{I}^{\mathbf{H}} \cdot \Delta_{I}^{\mathbf{H}}\right] d \mathbf{x}=\int_{K}\left[\underline{\underline{\varepsilon}} \frac{\partial}{\partial t} \Delta_{P}^{\mathbf{E}} \cdot \Delta_{I}^{\mathbf{E}}+\underline{\underline{\mu}} \frac{\partial}{\partial t} \Delta_{P}^{\mathbf{H}} \cdot \Delta_{I}^{\mathbf{H}}\right] d \mathbf{x} \\
& +\left[\int_{K} \underline{\underline{\varepsilon}} \frac{\partial}{\partial t} \pi_{h} \mathbf{E} \cdot \Delta_{I}^{\mathbf{E}} d \mathbf{x}-\int_{K}^{G} \underline{\varepsilon} \frac{\partial}{\partial t} \pi_{h} \mathbf{E} \cdot \Delta_{I}^{\mathbf{E}}\right]+\left[\int_{K} \underline{\mu} \frac{\partial}{\partial t} \pi_{h} \mathbf{H} \cdot \Delta_{I}^{\mathbf{H}} d \mathbf{x}\right. \\
& \left.-\int_{K}^{G} \underline{\underline{\mu}} \frac{\partial}{\partial t} \pi_{h} \mathbf{H} \cdot \Delta_{I}^{\mathbf{H}}\right]+\left[\int_{K} \underline{\underline{\sigma}} \pi_{h} \mathbf{E} \cdot \Delta_{I}^{\mathbf{E}} d \mathbf{x}-\int_{K}^{G} \underline{\underline{\sigma}} \pi_{h} \mathbf{E} \cdot \Delta_{I}^{\mathbf{E}}\right] \\
& +\left[\int_{K} \mathbf{J}_{s} \cdot \Delta_{I}^{\mathbf{E}} d \mathbf{x}-\int_{K}^{G} \mathbf{J}_{s} \cdot \Delta_{I}^{\mathbf{E}}\right]+\int_{\partial K} \beta \llbracket \Delta_{I}^{\mathbf{H}} \times \mathbf{n}_{K} \rrbracket \cdot \Delta_{I}^{\mathbf{E}} \\
& +\int_{\partial K} \beta \llbracket \pi_{h} \mathbf{H} \times \mathbf{n}_{K} \rrbracket \cdot \Delta_{I}^{\mathbf{E}}+\int_{\partial K} \gamma \llbracket \pi_{h} \mathbf{E} \times \mathbf{n}_{K} \rrbracket \cdot \Delta_{I}^{\mathbf{H}} \\
& +\int_{\partial K} \gamma \llbracket \Delta_{I}^{\mathrm{E}} \times \mathbf{n}_{K} \rrbracket \cdot \Delta_{I}^{\mathrm{H}}+\int_{K} \nabla \times \Delta_{I}^{\mathrm{H}} \cdot \Delta_{I}^{\mathrm{E}} d \mathbf{x}-\int_{K} \nabla \times \Delta_{I}^{\mathrm{E}} \cdot \Delta_{I}^{\mathrm{H}} d \mathbf{x} \\
& -\int_{K} \nabla \times \Delta_{P}^{\mathbf{H}} \cdot \Delta_{I}^{\mathrm{E}} d \mathbf{x}+\int_{K} \nabla \times \Delta_{P}^{\mathrm{E}} \cdot \Delta_{I}^{\mathbf{H}} d \mathbf{x} \\
& -\int_{K}^{G} \underline{\underline{\sigma}} \Delta_{I}^{\mathrm{E}} \cdot \Delta_{I}^{\mathrm{E}}+\int_{K} \underline{\underline{\sigma}} \Delta_{P}^{\mathrm{E}} \cdot \Delta_{I}^{\mathrm{E}} d \mathbf{x} .
\end{aligned}
$$

To obtain (4.41), we have used the fact that the stiffness and the jump integrals are exact for all the elements belonging to the approximate space $U_{h}$. Indeed, for the stiffness terms we have the classical result: Let $\mathbf{u}_{h}, \mathbf{v}_{h} \in U_{h}$; we have $\forall K \in \mathscr{T}_{h}$,

$$
\begin{gathered}
\int_{K}^{G} \nabla \times \mathbf{u}_{h} \cdot \mathbf{v}_{h}=\int_{\hat{K}}^{G}\left|J_{K}\right| \frac{D F_{K}}{J_{K}} \hat{\nabla} \times \hat{\mathbf{u}}_{h} \cdot D F_{K}^{*-1} \hat{\mathbf{v}}_{h} \\
=\operatorname{sign}\left(J_{K}\right) \int_{\hat{K}}^{G} \hat{\nabla} \times \hat{\mathbf{u}}_{h} \cdot \hat{\mathbf{v}}_{h} \\
=\operatorname{sign}\left(J_{K}\right) \int_{\hat{K}} \hat{\nabla} \times \hat{\mathbf{u}}_{h} \cdot \hat{\mathbf{v}}_{h} d \hat{\mathbf{x}}=\int_{K} \nabla \times \mathbf{u}_{h} \cdot \mathbf{v}_{h} d \mathbf{x} .
\end{gathered}
$$

To write the last line of (4.42) we use the fact that the Gauss formula used is exact for all the polynomials in $Q_{2 r+1}(\hat{K})$. This is why we have omitted the symbol $G$ (for "Gauss") in these integrals.

As regards the discrete energy norm of the first line, one shows easily that it is equivalent to the energy norm without numerical integration. Indeed, let $\mathbf{u}_{h} \in U_{h}$; then we have:

$$
\int_{K}^{G} \mathbf{u}_{h} \cdot \mathbf{u}_{h} d \mathbf{x}=\int_{\hat{K}}^{G}\left|J_{K}\right| D F^{-1} D F_{K}^{*-1} \hat{\mathbf{u}}_{h} \cdot \hat{\mathbf{u}}_{h} d \hat{x} .
$$

By using the estimates (3.3) and (3.5), we can write immediately these two inequalities:

$$
C_{1} \frac{\rho_{K}^{3}}{h_{K}^{2}} \int_{\hat{K}}^{G} \hat{\mathbf{u}}_{h} \cdot \hat{\mathbf{u}}_{h} d \hat{x} \leq \int_{K}^{G} \mathbf{u}_{h} \cdot \mathbf{u}_{h} d \mathbf{x} \leq C_{2} \frac{h_{K}^{7}}{\rho_{K}^{6}} \int_{\hat{K}}^{G} \hat{\mathbf{u}}_{h} \cdot \hat{\mathbf{u}}_{h} d \hat{x}
$$


where $C_{1}, C_{2}>0$ are independent of $K$ and $r$.

Now, as the Gauss formula is exact to the order $2 r+1$ when we use $(r+1)^{3}$ quadrature points, we have:

$$
\int_{\hat{K}}^{\mathrm{G}} \hat{\mathbf{u}}_{h} \cdot \hat{\mathbf{u}}_{h} d \hat{x}=\int_{\hat{K}} \hat{\mathbf{u}}_{h} \cdot \hat{\mathbf{u}}_{h} d \hat{x} .
$$

Returning to the cell $K$ of the mesh:

$$
\begin{gathered}
\int_{\hat{K}} \hat{\mathbf{u}}_{h} \cdot \hat{\mathbf{u}}_{h} d \hat{x}=\int_{\hat{K}} D F_{K}^{*} \mathbf{u}_{h} o F_{K} \cdot D F_{K}^{*} \mathbf{u}_{h} o F_{K} d \hat{x} \\
=\int_{\hat{K}}\left|J_{K}\right| \frac{D F_{K} D F_{K}^{*}}{\left|J_{K}\right|} \mathbf{u}_{h} o F_{K} \cdot \mathbf{u}_{h} o F_{K} d \hat{x} .
\end{gathered}
$$

Again using (3.3) and (3.5), one obtains the following inequalities:

$$
\frac{\rho_{K}^{6}}{C_{2} h_{K}^{7}} \int_{K} \mathbf{u}_{h} \cdot \mathbf{u}_{h} d \mathbf{x} \leq \int_{\hat{K}} \hat{\mathbf{u}}_{h} \cdot \hat{\mathbf{u}}_{h} d \hat{x} \leq \frac{h_{K}^{2}}{C_{1} \rho_{K}^{3}} \int_{K} \mathbf{u}_{h} \cdot \mathbf{u}_{h} d \mathbf{x}
$$

Combining (4.44) and (4.47), we get the wanted result i.e.,

$$
\frac{C_{1}}{C_{2}} \sigma_{K}^{9} \int_{K} \mathbf{u}_{h} \cdot \mathbf{u}_{h} d \mathbf{x} \leq \int_{K}^{G} \mathbf{u}_{h} \cdot \mathbf{u}_{h} d \mathbf{x} \leq \frac{C_{2}}{C_{1}} \sigma_{K}^{9} \int_{K} \mathbf{u}_{h} \cdot \mathbf{u}_{h} d \mathbf{x} .
$$

From this equivalence, one deduces the following result: The assumption of regularity of the mesh gives:

$$
C \sigma^{9} \int_{K}\left[\underline{\underline{\varepsilon}} \Delta_{I}^{\mathbf{E}} \cdot \Delta_{I}^{\mathbf{E}}+\underline{\underline{\mu}} \Delta_{I}^{\mathbf{H}} \cdot \Delta_{I}^{\mathbf{H}}\right] d \mathbf{x} \leq \int_{K}^{G}\left[\underline{\underline{\varepsilon}} \Delta_{I}^{\mathbf{E}} \cdot \Delta_{I}^{\mathbf{E}}+\underline{\underline{\mu}} \Delta_{I}^{\mathbf{H}} \cdot \Delta_{I}^{\mathbf{H}}\right] d \mathbf{x}
$$

where $C>0$ is independent of $K$ and $r$.

Now, we are going to estimate the first integration error of the second line of (4.41):

$$
\begin{gathered}
\int_{K} \underline{\underline{\varepsilon}} \frac{\partial}{\partial t} \pi_{h} \mathbf{E} \cdot \Delta_{I}^{\mathbf{E}} d \mathbf{x}-\int_{K}^{G} \underline{\underline{\varepsilon}} \frac{\partial}{\partial t} \pi_{h} \mathbf{E} \cdot \Delta_{I}^{\mathbf{E}}=\int_{K} \underline{\underline{\varepsilon}} \pi_{h} \mathbf{E}_{t} \cdot \Delta_{I}^{\mathbf{E}} d \mathbf{x} \\
-\int_{K}^{G} \underline{\underline{\varepsilon}} \pi_{h} \mathbf{E}_{t} \cdot \Delta_{I}^{\mathbf{E}}=\int_{K} \underline{\underline{\varepsilon}}\left(\pi_{h} \mathbf{E}_{t}-\mathbf{E}_{t}\right) \cdot \Delta_{I}^{\mathbf{E}} d \mathbf{x}+\int_{K} \underline{\underline{\varepsilon}} \mathbf{E}_{t} \cdot \Delta_{I}^{\mathbf{E}} d \mathbf{x} \\
-\int_{K}^{G} \underline{\underline{\varepsilon}} \mathbf{E}_{t} \cdot \Delta_{I}^{\mathbf{E}}+\int_{K}^{G} \underline{\underline{\varepsilon}}\left(I_{h} \mathbf{E}_{t}-\pi_{h} \mathbf{E}_{t}\right) \cdot \Delta_{I}^{\mathbf{E}} \\
\leq\left\|\pi_{h} \mathbf{E}_{t}-\mathbf{E}_{t}\right\|_{0, K, \underline{\underline{\varepsilon}}}\left\|\Delta_{I}^{\mathbf{E}}\right\|_{0, K, \underline{\underline{\varepsilon}}}+\left|\int_{K} \underline{\underline{\varepsilon}} \mathbf{E}_{t} \cdot \Delta_{I}^{\mathbf{E}} d \mathbf{x}-\int_{K}^{G} \underline{\underline{\varepsilon}} \mathbf{E}_{t} \cdot \Delta_{I}^{\mathbf{E}}\right| \\
+C\left\|\pi_{h} \mathbf{E}_{t}-I_{h} \mathbf{E}_{t}\right\|_{0, K, \underline{\underline{\varepsilon}}}\left\|\Delta_{I}^{\mathbf{E}}\right\|_{0, K, \underline{\underline{\varepsilon}}} \\
\leq(1+C)\left\|\pi_{h} \mathbf{E}_{t}-\mathbf{E}_{t}\right\|_{0, K, \underline{\underline{\varepsilon}}}\left\|\Delta_{I}^{\mathbf{E}}\right\|_{0, K, \underline{\underline{\varepsilon}}}+C\left\|I_{h} \mathbf{E}_{t}-\mathbf{E}_{t}\right\|_{0, K, \underline{\underline{\varepsilon}}}\left\|\Delta_{I}^{\mathbf{E}}\right\|_{0, K, \underline{\underline{\varepsilon}}} \\
+\left|\int_{K} \underline{\underline{\underline{\varepsilon}}} \mathbf{E}_{t} \cdot \Delta_{I}^{\mathbf{E}} d \mathbf{x}-\int_{K}^{G} \underline{\underline{\varepsilon}} \mathbf{E}_{t} \cdot \Delta_{I}^{\mathbf{E}}\right|
\end{gathered}
$$

where $C$ is independent of $K$ and $r$. 
We obtain the previous inequalities by combining the Schwarz discrete inequality and the previous equivalence property. To estimate the last line of (4.50), only for the last term, additional work is necessary. First, we develop this term:

$$
\begin{gathered}
\left|\int_{K} \underline{\underline{\varepsilon}} \mathbf{E}_{t} \cdot \Delta_{I}^{\mathbf{E}} d \mathbf{x}-\int_{K}^{G} \underline{\underline{\varepsilon}} \mathbf{E}_{t} \cdot \Delta_{I}^{\mathbf{E}}\right| \\
=\left|\int_{\hat{K}}\right| J_{K}\left|D F_{K}^{-1} \underline{\underline{\varepsilon}} O F_{K} D F_{K}^{*-1} \hat{\mathbf{E}}_{t} \cdot \hat{\Delta}_{I}^{\mathbf{E}} d \mathbf{x}-\int_{\hat{K}}^{G}\right| J_{K}\left|D F_{K}^{-1} \underline{\underline{\varepsilon}} O F_{K} D F_{K}^{*-1} \hat{\mathbf{E}}_{t} \cdot \hat{\Delta}_{I}^{\mathbf{E}}\right|
\end{gathered}
$$

where $\hat{\mathbf{E}}_{t}=D F_{K}^{*} \mathbf{E}_{t} o F_{K}$ and $\hat{\Delta}_{I}^{\mathbf{E}}=\hat{\mathbf{E}}_{h}-\hat{\pi}_{r}^{1} \hat{\mathbf{E}} \in\left[Q_{r}(\hat{K})\right]^{3}$.

Let $\hat{w}=\left|J_{K}\right| D F_{K}^{-1} \underline{\underline{\varepsilon}} o F_{K} D F_{K}^{*-1} \hat{\mathbf{E}}_{t}$. Introduce the interpolation polynomial $\hat{I}^{r} \hat{\mathbf{w}}$ in (4.51) and using the fact that the Gauss quadrature rule is exact for the polynomial space $Q_{2 r+1}$ when we take $(r+1)^{3}$ quadrature points, we get:

$$
\begin{aligned}
& \left|\int_{K} \underline{\underline{\varepsilon}} \mathbf{E}_{t} \cdot \Delta_{I}^{\mathbf{E}} d \mathbf{x}-\int_{K}^{G} \stackrel{\underline{\varepsilon}}{\underline{\mathbf{E}_{t}} \cdot \Delta_{I}^{\mathbf{E}}}\right|=\left|\int_{\hat{K}} \hat{\mathbf{w}} \cdot \hat{\Delta}_{I}^{\mathbf{E}} d \mathbf{x}-\int_{K} \hat{I}^{r} \hat{\mathbf{w}} \cdot \hat{\Delta}_{I}^{\mathbf{E}} d \mathbf{x}\right| \\
& =\left|\int_{\hat{K}}\left(\hat{\mathbf{w}}-\hat{I}^{r} \hat{\mathbf{w}}\right) \cdot \hat{\Delta}_{I}^{\mathbf{E}} d \mathbf{x}\right| \leq C\left\|\hat{\mathbf{w}}-\hat{I}^{r} \hat{\mathbf{w}}\right\|_{0, \hat{K}}\left\|\hat{\Delta}_{I}^{\mathbf{E}}\right\|_{0, \hat{K}, \underline{\underline{\varepsilon}}}
\end{aligned}
$$

where $C$ depends on $C_{1}$.

Using the Bramble-Hilbert lemma and the theory of the spectral methods [31, we can write the two following estimates for the interpolation operator $\hat{I}^{r}$ :

$$
\begin{gathered}
\left\|\hat{I}^{r}(\hat{\mathbf{w}})-\hat{\mathbf{w}}\right\|_{0, \hat{K}} \leq \frac{C(\hat{K})}{r^{r+1}}[\hat{\mathbf{w}}]_{r+1, \hat{K}}, \\
\left\|\hat{I}^{r}(\hat{\mathbf{w}})-\hat{\mathbf{w}}\right\|_{0, \hat{K}} \leq \frac{C}{r^{s}}\|\hat{\mathbf{w}}\|_{s, \hat{K}} .
\end{gathered}
$$

First, we are going to estimate the term $[\hat{\mathbf{w}}]_{r+1, \hat{K}}$. The definition of $\hat{\mathbf{w}}$ leads to:

$$
[\hat{\mathbf{w}}]_{r+1, \hat{K}}=\left[\left|J_{K}\right| D F_{K}^{-1} \underline{\underline{\varepsilon}} O F_{K} \mathbf{E}_{t} o F_{K}\right]_{r+1, \hat{K}}=\left[M_{K} \underline{\underline{\varepsilon}} O F_{K} \mathbf{E}_{t} o F_{K}\right]_{r+1, \hat{K}}
$$

where $M_{K}=\left(m_{i, j}^{K}\right) \in \mathscr{M}(3,3)$, the cofactor matrix of $D F_{K}$. Developing (4.54), we get:

$$
\begin{aligned}
& {\left[M_{K} \underline{\underline{\varepsilon}} o F_{K} \mathbf{E}_{t} o F_{K}\right]_{r+1, \hat{K}}^{2}=\sum_{i=1}^{3}\left[\sum_{j=1}^{3} m_{i, j}^{K} \sum_{k=1}^{3} \varepsilon_{j, k} \mathbf{E}_{t}^{k} o F_{K}\right]_{r+1, \hat{K}}^{2}} \\
& \leq 6 \sum_{i=1}^{3} \sum_{j=1}^{3} \sum_{k=1}^{3}\left[m_{i, j}^{K} \varepsilon_{j, k} \mathbf{E}_{t}^{k} o F_{K}\right]_{r+1, \hat{K}}^{2} \\
& \leq 6 \sum_{i=1}^{3} \sum_{j=1}^{3} \sum_{k=1}^{3} \sum_{l=1}^{3} \int_{\hat{K}}\left|\frac{\partial^{r+1}}{\partial \hat{x}_{l}^{r+1}}\left(m_{i, j}^{K} \varepsilon_{j, k} \mathbf{E}_{t}^{k} o F_{K}\right)\right|^{2} d \hat{\mathbf{x}} \\
& \leq 6 \sum_{i=1}^{3} \sum_{j=1}^{3} \sum_{k=1}^{3} \sum_{l=1}^{3} \varepsilon_{j, k} \int_{\hat{K}}\left|\sum_{m=0}^{r+1}\left(\begin{array}{c}
m \\
r+1
\end{array}\right) \frac{\partial^{m}}{\partial \hat{x}_{l}^{m}}\left(m_{i, j}^{K}\right) \frac{\partial^{r+1-m}}{\partial \hat{x}_{l}^{r+1-m}}\left(\mathbf{E}_{t}^{k} o F_{K}\right)\right|^{2} d \hat{\mathbf{x}}
\end{aligned}
$$

(because $\underline{\underline{\varepsilon}}$ is constant within a cell). 
It is easy to see that $\forall m \geq 3, \frac{\partial^{m}}{\partial \hat{x}_{k}^{m}}\left(m_{i, j}^{K}\right)=0$ and that $0 \leq m \leq 2,\left|\frac{\partial^{m}}{\partial \hat{x}_{k}^{m}}\left(m_{i, j}^{K}\right)\right| \leq$ $C h_{K}^{2}$. That implies the following estimate:

$$
\begin{gathered}
{\left[M_{K} \underline{\underline{\varepsilon}} o F_{K} \mathbf{E}_{t} o F_{K}\right]_{r+1, \hat{K}}^{2}} \\
\leq C \sum_{i=1}^{3} \sum_{j=1}^{3} \sum_{k=1}^{3} \sum_{l=1}^{3} \sum_{m=0}^{2} \int_{\hat{K}}\left|\frac{\partial^{m}}{\partial \hat{x}_{l}^{m}}\left(m_{i, j}^{K}\right) \frac{\partial^{r+1-m}}{\partial \hat{x}_{l}^{r+1-m}}\left(\mathbf{E}_{t}^{k} o F_{K}\right)\right|^{2} d \hat{\mathbf{x}} \\
\leq C h_{K}^{4} \sum_{i=1}^{3} \sum_{j=1}^{3} \sum_{k=1}^{3} \sum_{l=0}^{3} \sum_{m=0}^{2} \int_{\hat{K}}\left|\frac{\partial^{r+1-m}}{\partial \hat{x}_{l}^{r+1-m}}\left(\mathbf{E}_{k}^{j} o F_{K}\right)\right|^{2} d \hat{\mathbf{x}} .
\end{gathered}
$$

Finally, we have:

$$
[\hat{\mathbf{w}}]_{r+1, \hat{K}}^{2} \leq C h_{K}^{4}\left(\left[\mathbf{E}_{t} o F_{K}\right]_{r+1, \hat{K}}^{2}+\left[\mathbf{E}_{t} o F_{K}\right]_{r, \hat{K}}^{2}+\left[\mathbf{E}_{t} o F_{K}\right]_{r-1, \hat{K}}^{2}\right) .
$$

Now, using (3.28), we deduce the following three estimates:

$$
\begin{gathered}
{\left[\mathbf{E}_{t} o F_{K}\right]_{r+1, \hat{K}} \leq C h_{K}^{r-\frac{1}{2}}\left|\mathbf{E}_{t}\right|_{r+1, K},} \\
{\left[\mathbf{E}_{t} o F_{K}\right]_{r, \hat{K}} \leq C h_{K}^{r-\frac{3}{2}}\left|\mathbf{E}_{t}\right|_{r, K},} \\
{\left[\mathbf{E}_{t} o F_{K}\right]_{r-1, \hat{K}} \leq C h_{K}^{r-\frac{5}{2}}\left|\mathbf{E}_{t}\right|_{r-1, K} .}
\end{gathered}
$$

Injecting (4.58) in (4.57), we obtain:

$$
[\hat{\mathbf{w}}]_{r+1, \hat{K}} \leq C\left(h_{K}^{r+\frac{3}{2}}\left|\mathbf{E}_{t}\right|_{r+1, K}+h_{K}^{r+\frac{1}{2}}\left|\mathbf{E}_{t}\right|_{r, K}+h_{K}^{r-\frac{1}{2}}\left|\mathbf{E}_{t}\right|_{r-1, K}\right) .
$$

Moreover, we have:

$$
\left\|\hat{\Delta}_{I}^{E}\right\|_{0, \hat{K}} \leq \frac{C}{h_{K}^{\frac{1}{2}}}\left\|\Delta_{I}^{\mathbf{E}}\right\|_{0, K, \underline{\underline{\varepsilon}}} .
$$

Combining (4.59) and (4.60), we have the following error estimate for the interpolation operator:

$$
\begin{gathered}
\left|\int_{K}^{G} \mathbf{E}_{t} \cdot \Delta_{I}^{\mathbf{E}} d \mathbf{x}-\int_{K} \mathbf{E}_{t} \cdot \Delta_{I}^{\mathbf{E}} d \mathbf{x}\right| \leq \frac{C}{r^{r+1}}\left(h_{K}^{r+1}\left|\mathbf{E}_{t}\right|_{r+1, K}+h_{K}^{r}\left|\mathbf{E}_{t}\right|_{r, K}\right. \\
\left.+h_{K}^{r-1}\left|\mathbf{E}_{t}\right|_{r-1, K}\right)\left\|\Delta_{I}^{\mathbf{E}}\right\|_{0, K, \underline{\underline{\varepsilon}}}
\end{gathered}
$$

Finally, using (4.61) and (4.39), (4.50) gives: For $0<h_{K} \leq 1$,

$$
\left|\int_{K}^{G} \underline{\varepsilon} \pi_{h} \mathbf{E}_{t} \cdot \Delta_{E}^{I}-\int_{K} \underline{\varepsilon} \pi_{h} \mathbf{E}_{t} \cdot \Delta_{E}^{I} d \mathbf{x}\right| \leq C \frac{h_{K}^{r-1}}{r^{r+1}}\left\|\mathbf{E}_{t}\right\|_{r+1, K}\left\|\Delta_{E}^{I}\right\|_{0, K, \text { tens } \varepsilon} .
$$


Proceeding in the same way, we prove:

$$
\begin{aligned}
& \bullet\left|\int_{K}^{G} \underline{\underline{\mu}} \pi_{h} \mathbf{H}_{t} \cdot \Delta_{H}^{I}-\int_{K} \underline{\underline{\mu}} \pi_{h} \mathbf{H}_{t} \cdot \Delta_{H}^{I} d \mathbf{x}\right| \leq C \frac{h_{K}^{r-1}}{r^{r+1}}\left\|\mathbf{H}_{t}\right\|_{r+1, K}\left\|\Delta_{H}^{I}\right\|_{0, K, \underline{\underline{\varepsilon}}}, \\
& \bullet\left|\int_{K}^{G} \underline{\underline{\sigma}} \pi_{h} \mathbf{E} \cdot \Delta_{E}^{I}-\int_{K} \underline{\underline{\sigma}} \pi_{h} \mathbf{E} \cdot \Delta_{E}^{I} d \mathbf{x}\right| \leq C \frac{h_{K}^{r-1}}{r^{r+1}}\|\mathbf{E}\|_{r+1, K}\left\|\Delta_{E}^{I}\right\|_{0, K, \underline{\underline{\varepsilon}}}, \\
& \bullet\left|\int_{K}^{G} \mathbf{J}_{s} \cdot \Delta_{E}^{I}-\int_{K} \mathbf{J}_{s} \cdot \Delta_{E}^{I} d \mathbf{x}\right| \leq C \frac{h_{K}^{r-1}}{r^{r+1}}\left\|\mathbf{J}_{s}\right\|_{r+1, K}\left\|\Delta_{E}^{I}\right\|_{0, K, \underline{\underline{\varepsilon}}} .
\end{aligned}
$$

From (4.62) and (4.63), we deduce that it suffices to add the error (after the temporal integration from 0 to $T$ ):

$$
\begin{gathered}
C \frac{h^{\min \left(s-1, s^{\prime}-1, s^{\prime \prime}-1, r-1\right)}}{r^{\min \left(s+1, s^{\prime}+1, s^{\prime \prime}+1\right)}}\left(\operatorname { m a x } _ { t \in [ 0 , T ] } \left(\left\|\mathbf{E}_{t}\right\|_{s^{\prime}+1, h},\left\|\mathbf{H}_{t}\right\|_{s^{\prime}+1, h},\right.\right. \\
\left.\left.\|\mathbf{E}\|_{s+1, h},\left\|\mathbf{J}_{s}\right\|_{s^{\prime \prime}+1, h}\right)\right) T
\end{gathered}
$$

from $\frac{1}{2}<s, s^{\prime}, s^{\prime \prime} \leq r$ (one had $s, s^{\prime}, s^{\prime \prime} \geq 0$ when the numerical integration was not used) for the space error estimate using the mass-lumping technique. Here $C$ is a positive constant independent of $K$ and $r$.

We conclude that the use of the Gauss quadrature formula can generate a deterioration of the spatial convergence $\left(s^{\prime}-1, s^{\prime \prime}-1\right)$ when the exact solution of the problem is not very regular inside at least a cell. Nevertheless, if the data of the treated problem are regular, then the mass-lumping does not generate a deterioration of the $h$ convergence (i.e. $h_{K}^{r-1}$ ). Moreover, this reinforces the risk of inconsistency of the scheme using $r=1$. Lastly, the behavior remains linear in time.

\section{Numerical RESUlts}

The aim of this part is to numerically verify whether the $h$-convergence rates obtained in the previous sections are optimal or not. To carry out this purpose, we study the propagation of a mode inside a perfectly metallic cubic cavity $(\mathbf{E} \times \mathbf{n}=0$ on the wall of the cavity) with an edge of $a=0.25 \mathrm{~m}$. The propagative mode that we study is a mode $(m, n, 0)$ given by:

$$
\left\{\begin{array}{l}
E_{x}=0 ; E_{y}=0 ; H_{z}=0 \\
E_{z}=\sin \left(m \pi \frac{x}{a}\right) \sin \left(n \pi \frac{y}{a}\right) \cos (\omega t), \\
H_{x}=\frac{\pi n}{a \omega \mu_{0}} \sin \left(m \pi \frac{x}{a}\right) \cos \left(n \pi \frac{y}{a}\right) \sin (\omega t), \\
H_{y}=\frac{\pi m}{a \omega \mu_{0}} \cos \left(m \pi \frac{x}{a}\right) \sin \left(n \pi \frac{y}{a}\right) \sin (\omega t),
\end{array}\right.
$$

where $\omega=3 \cdot 10^{8} \sqrt{\left(\frac{m \pi}{a}\right)^{2}+\left(\frac{n \pi}{a}\right)^{2}}$.

By imposing this mode as an initial condition (i.e. for $t=0$ ), the DG scheme gives an approached solution of (5.1). Hence, one knows the exact solution of our 
problem. We can then compute the errors due to the DG scheme for some appropriated norms. More precisely, we have used two norms. The first is the classical $\mathrm{L}^{2}$ norm $\left(\|\cdot\|_{0, \Omega}\right)$ and the second is the norm

$$
\|\mathbf{u}\|_{h}^{2}=\|\mathbf{u}\|_{0, \Omega}^{2}+\sum_{K \in \mathscr{T}_{h}}\|\nabla \times \mathbf{u}\|_{0, K}^{2}+\sum_{\Gamma \in \mathscr{F}_{h}}\|\llbracket \mathbf{u} \times \mathbf{n} \rrbracket\|_{0, \Gamma}^{2}
$$

which gives the classical $H(\operatorname{curl}, \Omega)$ norm when $\mu$ is curl-conforming.

Moreover, we have chosen to take a sufficiently small time step in order to have a negligible time error.

Finally, we have used two types of mesh. The first is a "slightly deformed" cartesian type and the second is obtained by cutting each tetrahedron of a tetrahedrique mesh in four hexahedrons.

Remark 5.1. The simulations are carried out for the approximation $Q_{1}$ and $Q_{2}$. For highers orders, the computational cost rapidly becomes too important when one wants to obtain the asymptotic behaviour.

\section{a- "slightly deformed" Cartesian grids:}

The meshes are composed of $N \times N \times N$ cells, $N$ being the number of subdivisions in each direction. For the $Q_{1}$ and $Q_{2}$ approximations, we have respectively taken $m=n=1$ and $m=n=3$. We have determined both the projection errors (i.e. for $t=0$ ) and the DG errors obtained after having covered one period (i.e. $t=\frac{\omega}{2 \pi}$ ).

Tables 1 and 3 contain the results obtained for the $L^{2}$ projection of the initial condition. We find the theoretical rates, i.e., $h^{r}$ for the $L^{2}$ norm and $h^{r-1}$ for the norm $\|\cdot\|_{h}$. Indeed, the slight deformation has been made in order to obtain the estimation $\left|F_{K}\right|_{2, \infty, \hat{K}} \leq C h_{K}$ (and not $h_{K}^{2}$ ). Moreover, under this hypothesis, the theoretical results predicate that the $L^{2}$ error of the DG scheme is bounded by $O\left(h^{r-1}\right)$. However, the results contained in Tables 2 and 3 show that the $h$ convergence rates are $h^{r}$ for the norm $\|\cdot\|_{0, \Omega}$ and $h^{r-1}$ for the norm $\|\cdot\|_{h}$. To conclude, for this type of mesh, the theoretical convergence rates obtained seem to be sub-optimals.

TABle 1. Projection errors on "slightly deformed" Cartesian grids for $Q_{1}$.

\begin{tabular}{|c|c|c|c|c|}
\hline$Q_{1}$ & $8 \times 8 \times 8$ & $16 \times 16 \times 16$ & $32 \times 32 \times 32$ & $64 \times 64 \times 64$ \\
\hline \hline $\mathrm{L}^{2}$ error & $1.2812 \cdot 10^{-2}$ & $5.775 \cdot 10^{-3}$ & $2.835 \cdot 10^{-3}$ & $1.439 \cdot 10^{-3}$ \\
\hline $\mathrm{L}^{2}$ order & $\mathrm{X}$ & 1.14 & 1.026 & 0.98 \\
\hline$\|\cdot\|_{h}$ error & 0.1938 & 0.1754 & 0.172 & 0.1736 \\
\hline$\|\cdot\|_{h}$ order & $\mathrm{X}$ & $\approx 0$ & $\approx 0$ & $\approx 0$ \\
\hline
\end{tabular}

TABLE 2. Error after one period on "slightly deformed" Cartesian grids for $Q_{1}$.

\begin{tabular}{|c|c|c|c|c|}
\hline$Q_{1}$ & $8 \times 8 \times 8$ & $16 \times 16 \times 16$ & $32 \times 32 \times 32$ & $64 \times 64 \times 64$ \\
\hline \hline $\mathrm{L}^{2}$ error & $2.794 \cdot 10^{-2}$ & $1.611 \cdot 10^{-2}$ & $8.342 \cdot 10^{-3}$ & $4.189 \cdot 10^{-3}$ \\
\hline $\mathrm{L}^{2}$ order & $\mathrm{X}$ & 0.7 & 0.95 & 0.993 \\
\hline$\|\cdot\|_{h}$ error & 0.25 & 0.263 & 0.267 & 0.268 \\
\hline$\|\cdot\|_{h}$ order & $\mathrm{X}$ & $\approx 0$ & $\approx 0$ & $\approx 0$ \\
\hline
\end{tabular}


TABLE 3. Projection errors on "slightly deformed" Cartesian grids for $Q_{2}$.

\begin{tabular}{|c|c|c|c|}
\hline$Q_{2}$ & $16 \times 16 \times 16$ & $32 \times 32 \times 32$ & $64 \times 64 \times 64$ \\
\hline \hline $\mathrm{L}^{2}$ error & $1.708 \cdot 10^{-3}$ & $3.658 \cdot 10^{-4}$ & $8.772 \cdot 10^{-5}$ \\
\hline $\mathrm{L}^{2}$ order & $\mathrm{X}$ & 2.22 & 2.06 \\
\hline$\|\cdot\|_{h}$ error & $8.2951 \cdot 10^{-2}$ & $3.513 \cdot 10^{-2}$ & $1.675 \cdot 10^{-2}$ \\
\hline$\|\cdot\|_{h}$ order & $\mathrm{X}$ & 1.24 & 1.06 \\
\hline
\end{tabular}

TABLE 4. Error after one period on "slightly deformed" Cartesian grids for $Q_{2}$.

\begin{tabular}{|c|c|c|c|}
\hline$Q_{2}$ & $16 \times 16 \times 16$ & $32 \times 32 \times 32$ & $64 \times 64 \times 64$ \\
\hline \hline $\mathrm{L}^{2}$ error & $5.721 \cdot 10^{-3}$ & $1.206 \cdot 10^{-3}$ & $3.12 \cdot 10^{-4}$ \\
\hline $\mathrm{L}^{2}$ order & $\mathrm{X}$ & 2.24 & 1.95 \\
\hline$\|\cdot\|_{h}$ error & 0.16 & $5.947 \cdot 10^{-2}$ & $2.607 \cdot 10^{-2}$ \\
\hline$\|\cdot\|_{h}$ order & $\mathrm{X}$ & 1.42 & 1.18 \\
\hline
\end{tabular}

\section{b- General unstructured hexahedral meshes:}

For this numerical experiment, we have used meshes obtained by cutting each tetrahedron of a tetrahedrique mesh in four hexahedrons. We have taken an initial mesh that we have successively refined. As for the previous example, we have the estimation $\left|F_{K}\right|_{2, \infty, \hat{K}} \leq C h_{K}$. For the $Q_{1}$ and $Q_{2}$ approximations, we have taken $m=n=1$.

Tables [5]6 and 7, 8 contain the results obtained for the projection and the DG errors respectively. The first line of each table corresponds to the maximal spatial step $(h)$. For the projection, the convergence rates conform to the theoretical results i.e. $h^{r}$ for $\|\cdot\|_{0, \Omega}$ and $h^{r-1}$ for $\|\cdot\|_{h}$. With regard to the DG errors, the $Q_{1}$ and $Q_{2}$ approximations seem to have convergence rates for the $\mathrm{L}^{2}$ - norm equal to $h^{0}$ and $h^{1}$ respectively i.e. $h^{r-1}$. This result seems to be confirmed by the errors obtained for $\|\cdot\|_{h}$. Indeed for this norm, the convergence rates are $h^{-\alpha}$ for $Q_{1}$ and $h^{\beta}$ for $Q_{2}, \alpha$ and $\beta$ seemingly tending respectively towards 1 and 0 . For this type of mesh, the theoretical convergence rates seem to be optimal.

TABlE 5. Projection errors on general unstructured hexahedral meshes for $Q_{1}$.

\begin{tabular}{|c|c|c|c|}
\hline$Q_{1}$ & 0.039 & 0.021 & 0.011 \\
\hline \hline $\mathrm{L}^{2}$ error & $8.4622 \cdot 10^{-3}$ & $4.3179 \cdot 10^{-3}$ & $2.1568 \cdot 10^{-3}$ \\
\hline $\mathrm{L}^{2}$ order & $\mathrm{X}$ & 1.08 & 1.07 \\
\hline$\|\cdot\|_{h}$ error & 0.3575 & 0.3567 & 0.3544 \\
\hline$\|\cdot\|_{h}$ order & $\mathrm{X}$ & $\approx 0$ & $\approx 0$ \\
\hline
\end{tabular}


TABLE 6. Projection errors on general unstructured hexahedral meshes for $Q_{2}$.

\begin{tabular}{|c|c|c|c|}
\hline$Q_{2}$ & 0.078 & 0.039 & 0.021 \\
\hline \hline $\mathrm{L}^{2}$ error & $8.0761 \cdot 10^{-4}$ & $1.8373 \cdot 10^{-4}$ & $4.9105 \cdot 10^{-5}$ \\
\hline $\mathrm{L}^{2}$ order & $\mathrm{X}$ & 2.13 & 2.13 \\
\hline$\|\cdot\|_{h}$ error & $2.4309 \cdot 10^{-2}$ & $1.136 \cdot 10^{-2}$ & $5.8978 \cdot 10^{-3}$ \\
\hline$\|\cdot\|_{h}$ order & $\mathrm{X}$ & 1.09 & 1.05 \\
\hline
\end{tabular}

TABLE 7. Error after one period on general unstructured hexahedral meshes for $Q_{1}$.

\begin{tabular}{|c|c|c|c|}
\hline$Q_{1}$ & 0.039 & 0.021 & 0.011 \\
\hline \hline $\mathrm{L}^{2}$ error & $6.2637 \cdot 10^{-2}$ & $3.6486 \cdot 10^{-2}$ & $2.8541 \cdot 10^{-2}$ \\
\hline $\mathrm{L}^{2}$ order & $\mathrm{X}$ & 0.87 & 0.37 \\
\hline$\|\cdot\|_{h}$ error & 1.168 & 1.326 & 2.04 \\
\hline$\|\cdot\|_{h}$ order & $\mathrm{X}$ & -0.208 & -0.67 \\
\hline
\end{tabular}

TABLE 8. Error after one period on general unstructured hexahedral meshes for $Q_{2}$.

\begin{tabular}{|c|c|c|c|}
\hline$Q_{2}$ & 0.078 & 0.039 & 0.021 \\
\hline \hline $\mathrm{L}^{2}$ error & $6.6374 \cdot 10^{-3}$ & $2.6737 \cdot 10^{-3}$ & $1.1924 \cdot 10^{-3}$ \\
\hline $\mathrm{L}^{2}$ order & $\mathrm{X}$ & 1.31 & 1.3 \\
\hline$\|\cdot\|_{h}$ error & 0.1568 & 0.1152 & $9.8245 \cdot 10^{-2}$ \\
\hline$\|\cdot\|_{h}$ order & $\mathrm{X}$ & 0.44 & 0.25 \\
\hline
\end{tabular}

\section{REFERENCES}

1. Philippe G. Ciarlet, The finite element method for elliptic problems, North-Holland, 1978. MR0520174 (58:25001)

2. Achdou Yves, The Finite Element Methods, www.ann.jussieu.fr/achdou/enseignement.

3. Malika Remaki, Méthodes numériques pour les équations de Maxwell instationnnaires en Milieu hétérogène, Doctorat de Mathématiques Appliquées de l'Ecole Nationale des Ponts et Chaussées, 1999.

4. G. Cohen, Higher-order numerical methods for transient wave equations. Springer-Verlag, 2002. MR:1870851 (2002m:65069)

5. K.S. Yee, Numerical solution of initial boundary value problems involving Maxwell's equation in isotropic media. IEEE Trans. Antennas Prop., 14, 302-307, 1966.

6. A. Taflove (ed.), Advances in computational electrodynamics: The Finite-Difference TimeDomain, Artech House, Boston, 1998. MR1639352 (99c:78001)

7. Andreas C. Cangellaris and Diana B. Wright, Analysis of the Numerical Error Caused by the Stair-Stepped Approximation of a Conducting Boundary in FDTD Simulations of Electromagnetic Phenomena, IEEE Trans. Antennas Prop., vol. AP-39, No. 10, pp. 1518-1525, October 1991.

8. J.S. Hesthavens and T. Warburton, High-Order Nodal Methods on Unstructured Grids. I. Time-domain Solution of Maxwell's Equations, J. Comput. Phys., vol. 181, pp. 1-34, 2002. MR:1925981 (2003f:78034) 
9. Garry Rodrigue and Daniel White, A vector Finite Element Time-Domain Method for solving Maxwell's equations on unstructured hexahedral grids, SIAM J. Sci. Comput., vol. 23, No. 3, pp. 683-706, 2001. MR1860960 (2002h:78036)

10. P. Bonnet and X. Ferrieres, Numerical modeling of scattering problems using a time domain finite volume method, JEWA, vol. 11, pp. 1165-1189, 1997.

11. S. Piperno and M. Remaki, and L. Fezoui, A non-diffusive finite volume scheme for the $3 D$ Maxwell equations on unstructured meshes, SIAM J. Numer. Anal., vol. 39, No. 6, pp. 2089-2108, 2002. MR.1897951 (2003e:65153)

12. Bernardo Cockburn, Fengyan Li, and Chi-Wang Shu, Locally divergence-free discontinuous Galerkin methods for the Maxwell equations, J. of Comput. Phys., vol. 194, pp. 588-610, 2004. MR2034859 (2004j:78024)

13. Bernardo Cockburn and Chi-Wang Shu, The Runge-Kutta Discontinuous Galerkin Method for conservation law V, J. Comput. Phys., vol. 141, pp. 199-224, 1998. MR1619652 (99c:65181)

14. B. Cockburn, G.E. Karniadakis, and C-W. Shu, The Development of Discontinuous Galerkin Methods, Lecture Notes in Computational Science and Engineering, vol. 11, Springer, 2000. MR.1842161(2002e:65002)

15. Nicolas Canouet, Méthodes de Galerkin Discontinu pour la résolution du système de Maxwell sur des maillages localement raffinés non-conforme,Doctorat de Mathématiques Appliquées de l'Ecole Nationale des Ponts et Chaussées, December 2003.

16. Paul Houston, Ilaria Perugia, and Dominik Shötzau, Mixed Discontinuous Galerkin approximation of the Maxwell operator, SIAM J. Numer. Anal., vol. 42, No. 1, pp. 434-459, 2004. MR 2051073 (2005b:65128)

17. I. Perugia, D. Schötzau, and P. Monk, Stabilized interior penalty methods for timeharmonic Maxwell equations, Comput. Methods Appl. Mech. Eng., 191 (2002), pp.4675-4697. MR.1929626 (2003j:78058)

18. W. Reed and T. Hill, Triangular mesh methods for the neutron transport equation, Tech. Report LA-UR-73-479, Los Alamos National Laboratory, Los Alamos, New Mexico, USA, 1973.

19. P. Lesaint and P. Raviart, On a finite element method for solving the neutron transport equation, in Mathematical Aspects of Finite Element Methods in Partial Differential Equations, C. deBoor, ed., Academic Press, New York, 1974, pp. 89-123. MR0658142 (58:31918)

20. C. Johnson and J. Pitkäranta, An analysis of the discontinuous Galerkin method for a scalar hyperbolic equation, Math. Comp. 46 (1986), pp. 1-26. MR.815828 (88b:65109)

21. J.-C. Nédélec, Mixed finite elements in $\mathbb{R}^{3}$, Numer. Math., 35(3), pp. 315-341, 1980. MR592160 (81k:65125)

22. J.-C. Nédélec, A new family of mixed finite elements in $\mathbb{R}^{3}$, Numer. Math, 50(1), pp. 57-81, 1986. MR864305 (88e:65145)

23. G. Cohen and P. Monk, Mur-Nedelec finite element schemes for Maxwell's equations, Comp. Meth. in Appl. Mech. Eng., 169(3-4), pp. 197-217, 1999. MR.1675684 (99k:78002)

24. Peter Monk and Gerald R. Richter, A discontinuous Galerkin method for linear symmetric hyperbolic systems in inhomogeneous media. J. Sci. Comp. 22/23 (2005), 443-477. MR 2142205 (2006b:65144)

25. S. M. Rao, Time domain electromagnetics, Series Editor, David Irwin, Auburn University, Academic Press, 1999.

26. J. Jin, The finite Element Method in Electromagnetics, John Wiley \& Sons, New York, 1993. MR1903357 (2004b:78019)

27. S. Pernet, X. Ferrieres, and G. Cohen, An original finite element method to solve Maxwell's equations in time domain, Proceedings of EMC Zurich'2003, 18-20 February 2003, Zurich, Switzerland.

28. S. Prudhomme, F. Pascal, T. Oden, and A. Romkes, Review of a priori error estimation for Discontinuous Galerkin, Orsay, 2000, 2000-02.

29. B. Rivière, M.F. Wheeler, and V. Girault, A priori error estimates for finite element methods based on discontinuous approximation spaces for elliptic problems, SIAM J. Numer. Anal., 2001, 39, 3, pp. 902-931. MR1860450 (2002g:65149)

30. C. Bernardi and Y. Maday, Spectral Methods, in Handbook of Numerical Analysis, vol. V by P.G. Ciarlet and J.L. Lions (eds.), Elsevier Sciences, North-Holland, Amsterdam, 1997. MR 1470226 
31. D. Gottlieb and J.S. Hesthaven, Spectral methods for time-dependent problems, Cambridge Press.

32. A. Elmkies, Sur les éléments finis d'arête pour la résolution des équations de Maxwell en milieu anisotrope et pour des maillages quelconques, Université Paris IX-Dauphine, 1998, Thèse de mathématiques appliquées à l'ingéniérie.

33. V. Girault and P-A. Raviart, Finite element methods for Navier-Stoke equations, 1986, Springer-Verlag, New York. MR 851383 (88b:65129)

34. S. Pernet, Etude de méthodes d'ordre élevé pour résoudre les équations de Maxwell dans le domaine temporel. Application à la détection et à la compatibilité électromagnétique, Thesis, University of Paris, IX, November 2004.

Cerfacs (European Centre for Research and Advanced Training in Scientific Computation) 42, Avenue Gaspard Coriolis, 31057 Toulouse Cedex 01, France

E-mail address: pernet@cerfacs.fr

Onera, 2 avenue Edouard Belin, 31055 Toulouse, France

E-mail address: ferrieres@onecert.fr 